\title{
Nonlinear theory of a free electron laser with a helical wiggler and an axial guide magnetic field
}

\author{
N. S. Ginzburg and N. Yu. Peskov* \\ Institute of Applied Physics, Russian Academy of Sciences, Nizhny Novgorod 603950, Russia
}

(Received 27 March 2013; published 12 September 2013)

\begin{abstract}
A 1D nonlinear theory of a free electron laser (FEL) with a helical wiggler and an axial guide magnetic field is developed based on averaged equations of the electron motion. By averaging we separated two different cases of the e-beam/rf-wave interaction. The first one corresponds to the traditional wiggler synchronism (resonance) of $\mathrm{rf}$ wave with the electrons moving along stationary helical trajectories. The second one corresponds to combination resonances distinguishing by excitation of oscillation of the electrons near the stationary helical trajectory. Comparative analysis of the FEL operation in different regimes has been studied under the traditional wiggler synchronism condition. It was shown that FELs operated far from cyclotron resonance (including a reversed guide field orientation) possess low sensitivity to the initial velocity spread in the driving beam resulting in high electron efficiency. In contrast, under the weak guide field (the gyrofrequency is less than the bounce frequency) of a conventional orientation, the FEL efficiency is restricted by a significant increase in the transverse velocity of the electrons during the interaction with the $\mathrm{rf}$ wave that results in violation of the synchronism conditions and is accompanied by electron current losses. An additional mechanism of FEL efficiency enhancement under the conventional guide field orientation in the conditions when the gyrofrequency is higher than the bounce frequency, based on the dependence of the effective mass of the oscillating electrons on their energy, was demonstrated. Results of the theoretical analysis are compared with the results of experimental studies of FEL oscillators. The specific features of energy extraction from the electron beam under condition of an abnormal Doppler effect in the case of the combination resonance are described. This regime is beneficial to increase radiation frequency keeping wiggler period and electron energies.
\end{abstract}

DOI: 10.1103/PhysRevSTAB.16.090701

PACS numbers: 41.60.Cr, 52.59.Rz, 42.55.Tv, 84.40.Ik

\section{INTRODUCTION}

Free electron lasers (FELs) with a guide magnetic field are well established sources of powerful pulsed radiation of millimeter and submillimeter wavelengths bands. Experimental studies of such devices [1-12] have demonstrated effective operation with multimegawatt power level at millimeter wavelengths under high Doppler frequency up-shift.

In development of previous experimental results several successful experiments have been carried out recently exploiting different configurations of electron beams and wiggler fields [13-18]. In [13,14] FEL based on planar wiggler and driven by a large-size sheet electron beam was studied. In this experiment a two-dimensional (2D) distributed feedback realized by $2 \mathrm{D}$ Bragg resonators has been used for spatial synchronization of the radiation. In [14-16], a novel feedback mechanism has been applied to the coaxial scheme of FEL with magnetically

\footnotetext{
*Corresponding author. peskov@appl.sci-nnov.ru
}

Published by the American Physical Society under the terms of the Creative Commons Attribution 3.0 License. Further distribution of this work must maintain attribution to the author( $(s)$ and the published article's title, journal citation, and DOI. guided tubular electron beam and axial symmetric wiggler.

A more traditional FEL scheme driven by a pencil-like electron beam oscillating in a helical wiggler and an axial guide magnetic field has been realized in $[17,18]$ based on linear induction accelerator LIU-3000 (JINR, Dubna, Russia). This FEL operates at Ka band with output power at the level of $\sim 20-30 \mathrm{MW}$ and high electron efficiency (up to 25\%-30\%). Narrow spectrum width and stability of the single-mode generation regime allow application of this device for testing high-gradient accelerating structures [19]. In accordance with previous experimental studies [1-12], it was observed that performance of FELs of the mentioned type has strong dependence on strength and orientation of the guide magnetic field. In particular, electron efficiency both for amplifier [6,7] and oscillator $[17,18]$ schemes in regimes with a reversed guide magnetic field remarkably exceeds efficiency in the case of a conventional guide field orientation.

The high potential of the FEL with a guide magnetic field encouraged rather intensive theoretical studies during the past three decades [20-31]. The operating regime in this type of a FEL is determined by the ratio between the bounce frequency $\Omega_{b}=2 \pi v_{\|} / d_{w}$ of the forced particles oscillation in the helical wiggler field $\left(d_{w}\right.$ is a wiggler period and $v_{\|}=\beta_{\|} c$ is the particles axial velocity) and 
the gyrofrequency $\omega_{H}=e H_{0} / \gamma m c$ of its eigenoscillations in the uniform axial magnetic field $H_{0}$ as well as by the conditions required to pump transverse oscillations in the tapered wiggler section. Obviously, for an effective energy extraction from the electron beam, one needs to provide a particle motion with relatively constant axial velocity during the rf interaction. It may be achieved when an adiabatically tapered entrance for wiggler field is used. The adiabatic condition for the length of the wiggler entrance $l_{i n}$ has the form

$$
\left|\left(\omega_{H}-\Omega_{b}\right) \frac{l_{\text {in }}}{v_{\|}}\right| \gg 2 \pi .
$$

It should be noted that an adiabatic wiggler field entrance is optimal for intense relativistic electron beam transportation and utilized in the majority of FEL experiments with a guide magnetic field [1-19]. Under this condition in the tapered wiggler section the forced oscillations on the bounce frequency (bounce oscillations) are mainly excited in the electron beam with minimal admixture of "parasitic" eigenoscillations on the gyrofrequency. As a result, in the regular wiggler section, where the wiggler field amplitude becomes constant, the electrons move along stationary helical trajectories with a constant axial velocity or oscillate around this trajectory with the frequency $\Omega$ given by Eqs. (14) and (15) (see below). In this region the electrons are able to generate the rf wave of the frequency $\omega$ and the longitudinal wave number $h$ under the traditional wiggler resonance,

$$
\omega-h v_{\|} \approx \Omega_{b},
$$

which were observed in all mentioned experiments and studied in the most previous theoretical works [2,20-27]. At the same time the generation under the additional combination resonances

$$
\omega-h v_{\|} \approx \Omega_{b} \pm \Omega
$$

is also possible [28,29]. The radiation under condition (3) is accompanied by excitation of the small oscillations of electrons at the frequency $\Omega$ in the vicinity of the equilibrium stationary helical trajectories that is typical for an abnormal Doppler effect [32].

The present paper is devoted to the nonlinear theory of FELs with a guide magnetic field. Comprehensive analysis of the electron trajectories in the combined wiggler and axial guide magnetic field in the frame of both $1 \mathrm{D}$ and $3 \mathrm{D}$ models was carried out in [22-27]. Based on such trajectory analysis we extended this theory to describe the nonlinear stage of the interaction. We should emphasis that such an approach to derivation of the average motion equations, which is used in our paper, is originated from the theory of cyclotron resonance masers [33,34]. The averaged equations in the presence of the rf wave are obtained under the assumption that nonperturbed motion of electrons in the wiggler field takes place along stationary helical trajectories or near such a trajectory. The method of variation of arbitrary constants in equations which describe helical trajectory under the action of rf field is used. As a result, the consistent 1D nonlinear theory of FEL with guide magnetic field has been developed and averaged nonlinear equations for the electron-wave interaction under resonance conditions (2) and (3) have been obtained. We should note that previous theoretical investigations of the nonlinear dynamics of this type of an FEL are performed mainly by using a computer simulation of the full (nonaverage) equations of the electron-wave interaction or based on some simplified physical consideration (assumption of relatively small changes of electron energies, large detuning from the cyclotron resonance, etc.). In our paper we propose a more regular and universal method for derivation of the full motion equations that can be valid, in particular, not only for regimes with small changes of electron energies but in the regimes with high electron efficiency as well. The proposed average approach makes it possible to describe specific features of the rf interaction for different FEL operating regimes and to explain some experimental facts.

This paper is organized as follows. The motion of a magnetically guided electron beam in the tapered helical wiggler section is discussed in Sec. II. In Sec. III the proposed averaged approach to FEL's equations is described. In Sec. IV linear and nonlinear stages of the rf interaction under the conditions of the wiggler resonance (2) are studied for the different regimes of FEL operation determined by the ratio between the bounce frequency and gyrofrequency of the electrons. Results of the theoretical analysis are compared with the data of the linac driven FEL-oscillator experiments [17-19]. For combination resonances (3) the specific features of energy extraction from the electron beam under the condition of an abnormal Doppler effect are described in Sec. V. The possibility to advance the FEL into short-mm wavelengths exploiting combination resonance (3) is discussed. In the Appendix some details of the averaging procedure are presented.

\section{ELECTRONS MOTION IN THE PUMPING WIGGLER SECTION}

Let us consider a thin pencil-like electron beam moving near the axis of a helical wiggler where the magnetic field is circularly polarized and may be presented as

$$
\vec{H}_{w}=H_{w}(z)\left[\vec{x}_{0} \cos \left(h_{w} z\right)+\vec{y}_{0} \sin \left(h_{w} z\right)\right],
$$

where $h_{w}=2 \pi / d_{w}$ and $H_{w}(z)$ is amplitude of the wiggler field, which is smoothly varying along the axial $z$ coordinate to describe tapering of the field at the wiggler entrance. We assume that the radius of the transverse oscillations of the electrons $r_{b}=v_{\perp} / \Omega_{b}$ is relatively small: 


$$
h_{w} r_{b}=\frac{\beta_{\perp}}{\beta_{\|}} \ll 1
$$

( $v_{\perp}=\beta_{\perp} c$ is the particles transverse velocity). In this case we can neglect the transverse inhomogeneity of the wiggler field and adopt the 1D model for the electron trajectories (compare with [27]). Motion of the beam electrons in the wiggler field (4) and a homogeneous axial guide magnetic field $\vec{H}_{0}=H_{0} \vec{z}_{0}$ is described by the equations

$\frac{\mathrm{d} p_{+}}{\mathrm{d} Z}-\mathrm{i} f \frac{p_{+}}{p_{\|}}=-\mathrm{i} \alpha_{w} \mathrm{e}^{\mathrm{i} Z}, \quad \frac{\mathrm{d} p_{\|}}{\mathrm{d} Z}=\operatorname{Re}\left(\mathrm{i} \frac{p_{+}}{p_{\|}} \alpha_{w} \mathrm{e}^{\mathrm{i} Z}\right)$,

where the dimensionless variables are used: $Z=h_{w} z, p_{+}=$ $\left(p_{x}+\mathrm{i} p_{y}\right) / m c, p_{\|}=p_{z} / m c, p_{x, y, z}$ are the Cartesian components of the electrons momentum, $\alpha_{w}=e H_{w} / h_{w} m c^{2}$ and $f=\omega_{H 0} / h_{w} c, \omega_{H 0}=e H_{0} / m c$ is nonrelativistic gyrofrequency.

Taking into account the conservation of a particle kinetic energy in a magnetic field,

$$
E=\gamma m c^{2}=m c^{2} \sqrt{1+p_{\|}^{2}+\left|p_{+}\right|^{2}}=\text { const, }
$$

and representing the solution of (6) in the form $p_{+}=$ $\left(p_{1}+\mathrm{i} p_{2}\right) \mathrm{e}^{\mathrm{i} Z}$, where $p_{1,2}$ are real, we get the following system of equations:

$$
\begin{aligned}
& \frac{\mathrm{d} p_{1}}{\mathrm{~d} Z}=p_{2}\left(1-\frac{f}{\sqrt{\gamma^{2}-1-p_{1}^{2}-p_{2}^{2}}}\right), \\
& \frac{\mathrm{d} p_{2}}{\mathrm{~d} Z}=-\alpha_{w}-p_{1}\left(1-\frac{f}{\sqrt{\gamma^{2}-1-p_{1}^{2}-p_{2}^{2}}}\right) .
\end{aligned}
$$

Equation (7) can also be presented in the canonical form,

$$
\frac{\mathrm{d} p_{1}}{\mathrm{~d} Z}=-\frac{\partial \mathcal{H}}{\partial p_{2}}, \quad \frac{\mathrm{d} p_{2}}{\mathrm{~d} Z}=\frac{\partial \mathcal{H}}{\partial p_{1}},
$$

with the Hamiltonian

$\mathcal{H}=-\alpha_{w} p_{1}-\frac{p_{1}^{2}+p_{2}^{2}}{2}-f \sqrt{\gamma^{2}-1-p_{1}^{2}-p_{2}^{2}}$.

In the regular wiggler section, where the wiggler field amplitude is constant $\alpha_{w}=$ const, the equation $\mathcal{H}=$ const determines the particle orbits on the phase plane $\left(p_{1} ; p_{2}\right)$ (see Fig. 1). Equilibrium state $p_{1,2 *}$,

$$
p_{1 *}\left(1-\frac{f}{\sqrt{\gamma^{2}-1-p_{1 *}^{2}}}\right)=-\alpha_{w}, \quad p_{2 *}=0
$$

corresponds to the electron motion in the wiggler along the steady-state helical trajectory.

The dependence of the transverse momentum at the steady-state trajectory $p_{\perp *} \equiv p_{1 *}$ on the magnitude of the axial magnetic field $f$ is given in Fig. 2. For the strong axial magnetic fields $f>f_{c r}$ (i.e. when the gyrofrequency (a)

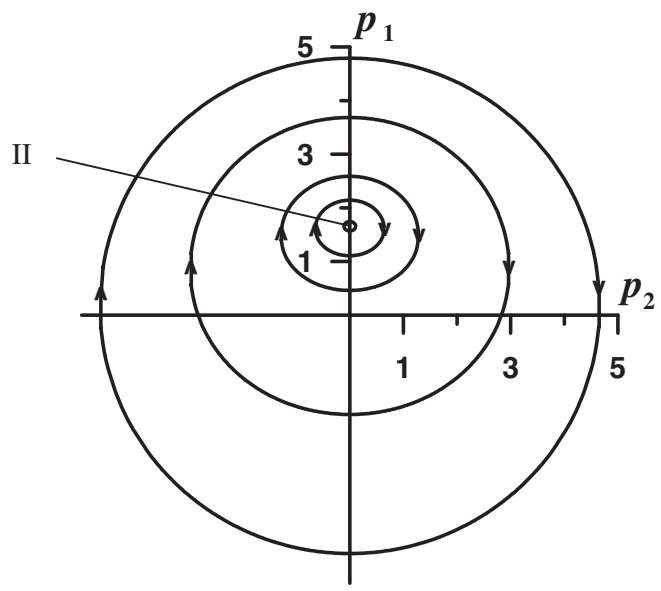

(b)

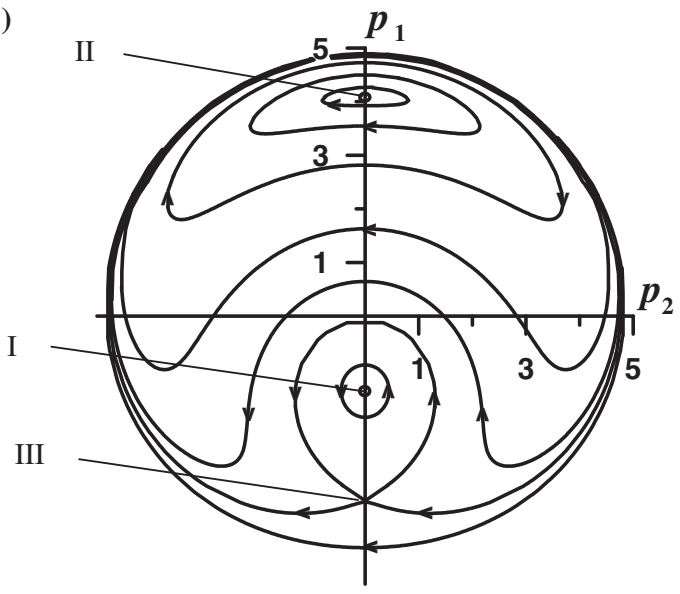

FIG. 1. The electron orbits on the phase plane $\left(p_{1}, p_{2}\right)$ at the different strengths of guide magnetic field: (a) $f>f_{c r}$ and (b) $f<f_{c r}\left(\gamma=5, \alpha_{w}=0.1\right)$.

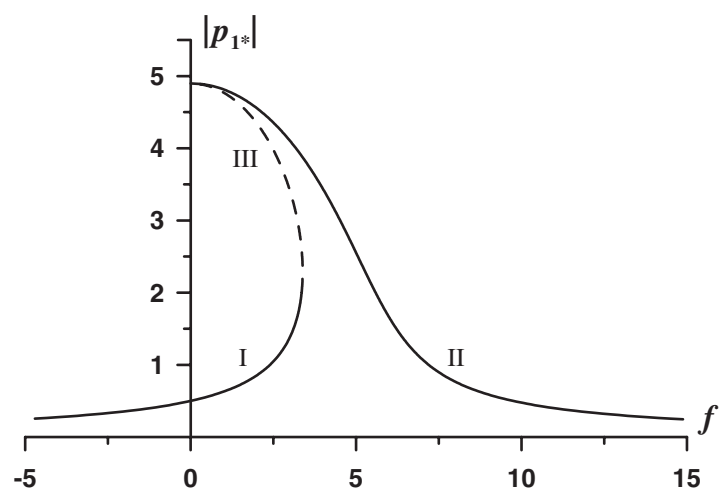

FIG. 2. The absolute value of electron transverse momentum $p_{\perp *}$ at the equilibrium helical trajectory as a function of guide magnetic field strength $f\left(\gamma=5, \alpha_{w}=0.1\right)$.

$\omega_{H}$ exceeds the bounce frequency $\Omega_{b}$ ), the electrons have only one equilibrium state [see Fig. 1(a)] while for weak axial magnetic fields $f<f_{c r}$ (i.e. $\omega_{H}<\Omega_{b}$ ) there exist three equilibrium states. The bifurcation value of the axial magnetic field 


$$
f_{c r}=\sqrt{\gamma^{2}-1}\left(1-\sqrt[3]{\frac{\alpha_{w}^{2}}{\gamma^{2}-1}}\right)^{\frac{3}{2}}
$$

can be found from (10) under the condition $\mathrm{d} f / \mathrm{d} p_{1 *}=0$.

To determine stability of the equilibrium states (10), let us introduce the minor perturbations $\rho_{1,2}$ as

$$
p_{1}=p_{1 *}+\rho_{1}, \quad p_{2}=\rho_{2},
$$

and assuming $\rho_{1,2} \ll p_{\perp *}$ linearize Eq. (7):

$$
\frac{\mathrm{d} \rho_{1}}{\mathrm{~d} Z}=\rho_{2}\left(1-\frac{f}{p_{\| * *}}\right), \quad \frac{\mathrm{d} \rho_{2}}{\mathrm{~d} Z}=-\rho_{1}\left(1-\frac{f}{p_{\| * *}}-\frac{f p_{\perp *}^{2}}{p_{\| *}^{3}}\right),
$$

where $p_{\| *}=\sqrt{\gamma^{2}-1-p_{\perp *}^{2}}$ is the axial momentum at the steady-state trajectory. Equation (13) is of a harmonic oscillator with the oscillation frequency:

$$
\Gamma=\sqrt{\left(1-\frac{f}{p_{\| *}}\right)\left(1-\frac{f}{p_{\| *}}-\frac{f p_{\perp *}^{2}}{p_{\| *}^{3}}\right)} .
$$

According to relations (10) and (14), stable equilibrium states (centers, $\Gamma^{2}>0$ ) belong to curves I and II in Fig. 2 (following [27] we will refer to these equilibrium states as groups of stationary orbits I and II), while unstable saddles $\left(\Gamma^{2}<0\right)$ take place on curve III (compare with Fig. 1).

Thus, Eq. (14) defines the frequency of electron oscillation around the helical stationary trajectory,

$$
\Omega=h_{w} v_{\|} \Gamma \text {. }
$$

In the case when the gyrofrequency and bounce frequency are sufficiently shifted apart from each other and when the wiggler field amplitude is small as

$$
\frac{\alpha_{w}^{2} f}{\left|\sqrt{\gamma^{2}-1}-f\right|^{3}} \ll 1,
$$

the relation (14) may be reduced to

$$
\Gamma=\left|1-\frac{f}{\sqrt{\gamma^{2}-1}}\right|\left(1-\frac{\alpha_{w}^{2} f}{2\left(\sqrt{\gamma^{2}-1}-f\right)^{3}}\right) .
$$

In the dimension term it means that the frequency of electron oscillation around the helical stationary trajectory is in fact the beat frequency between the gyro-oscillation and bounce oscillation:

$$
\Omega \approx\left|\Omega_{b}-\omega_{H}\right| .
$$

Let us now consider the process of pumping the transverse oscillations for the electrons in a smoothly uptapered wiggler section. If the condition of adiabaticity is fulfilled, i.e.

$$
\left|\frac{\Lambda}{\alpha_{w}} \frac{\mathrm{d} \alpha_{w}}{\mathrm{~d} Z}\right| \ll 1
$$

where $\Lambda=2 \pi / \Gamma$ is the period of electron oscillations in the phase plane, then the area embraced by the electron phase trajectory is preserved:

$$
\mathcal{J}=\oint p_{1} \mathrm{~d} p_{2}=\text { const. }
$$

We assume that at the wiggler entrance $z=0$, where the wiggler field tends to zero, the electrons move along the wiggler axis, i.e., in the variables used they are in the equilibrium state $\left(p_{1 *}=0 ; p_{2 *}=0\right)$ and $\mathcal{J}=0$. Then, with the increase in the wiggler field amplitude $\alpha_{w}$ due to the adiabatical invariant preservation (19), the electrons stay in the equilibrium state, which shifts along the ordinate (i.e. along the $p_{1}$ axis) in the plane $\left(p_{1} ; p_{2}\right)$. This corresponds to the excitation of the electron oscillation at the bounce frequency. For a strong axial magnetic field $f>$ $\sqrt{\gamma^{2}-1}$ (i.e. $f>f_{c r}$ at $\alpha_{w}=0$ ) when there is a single equilibrium state II positioned on the positive semiaxis $p_{1 *}>0$ [Fig. 1(a)], the above process of pumping the bounce oscillations takes place up to any value of $\alpha_{w}$. In contrast, for a weak magnetic field $f<\sqrt{\gamma^{2}-1}$ bounce oscillations are also excited for the electrons with the growth of $\alpha_{w}$ but only at the initial stage. In this case, on the phase plane the electrons are in the equilibrium state I which are positioned on the negative semiaxis $p_{1 *}<0$ [Fig. 1(b)]. However, increase in the wiggler field results in decrease of the parameter $f_{c r}$ [see formula (11)] and, finally, when the wiggler field reaches the critical value,

$$
\alpha_{c r}=\sqrt{\gamma^{2}-1-\sqrt[3]{f^{2}\left(\gamma^{2}-1\right)^{2}}}\left(1-\sqrt[3]{\frac{f}{\gamma^{2}-1}}\right),
$$

the condition $f=f_{c r}$ is met. At this point the bifurcation resulting in the confluence of the center and the saddle takes place on the phase plane. As a result, the condition of adiabaticity is broken because the period of particles motion $\Lambda$ in the vicinity of the separatrix tends to infinity. With the further increase in the wiggler field, when $\alpha_{w}$ becomes higher than the critical value given by (20), both the center and the saddle vanish and the phase plane becomes identical to that shown in Fig. 1(a). In this region of $\alpha_{w}$ on the phase plane, the electrons move along the trajectory embracing the area on the phase plane equal to that under the separatrix at the point of bifurcation. The motion of the electrons in real space becomes biperiodical with variable axial velocity and represents a combination of bounce oscillation and gyro-oscillation. ${ }^{1}$

If the electrons possess cyclotron oscillations before they entered the wiggler (i.e. possess nonzero initial pitch angle at the wiggler entrance) $p_{1,2}(z=0) \neq 0$, then these

\footnotetext{
${ }^{1}$ When the wiggler field would be spatially switched off, the electrons acquire only the cyclotron oscillations. Using the described mechanism, the method of a high-quality helical electron beam formation for the cyclotron autoresonance masers (CARMs) can be realized.
} 
oscillations will be preserved during the motion within the wiggler as well. On the phase plane it corresponds to small particle oscillations near the equilibrium state. The amplitude of these oscillations can be found using the condition of preservation of the phase area at the initial $(z=0)$ and final $\left(z=l_{\text {in }}\right)$ stages.

Nonadiabaticity of the wiggler field tapering can be considered as another possible reason for cyclotron oscillation excitation near the equilibrium states. To prevent excitation of parasitic cyclotron oscillations, the length of the tapered wiggler section should be increased when $\omega_{H}$ and $\Omega_{b}$ approach one another [as it follows from condition (1)].

Figures 3 and 4 give the results of numerical simulation of Eq. (7) for particle motions in the tapered wiggler section

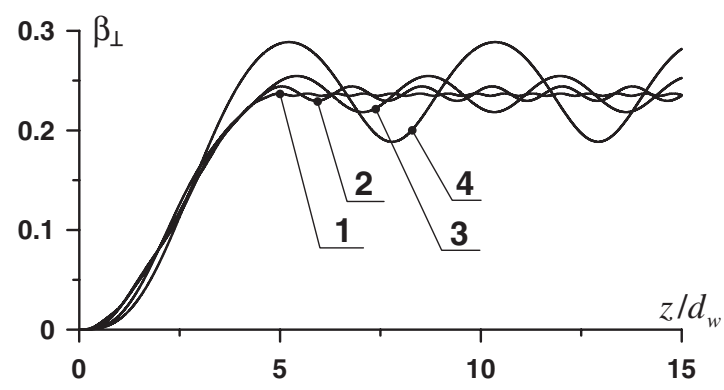

FIG. 3. Trajectory of electrons $\beta_{\perp}(z)$ in the wiggler with uptapered entrance for different guide field strength $f$ : (1) $f / f_{c r}=-0.4, \quad \alpha_{w}=1.0 ;$ (2) $f / f_{c r}=0.4, \quad \alpha_{w}=0.4$; (3) $f / f_{c r}=0.65, \alpha_{w}=0.25$; (4) $f / f_{c r}=0.85, \alpha_{w}=0.15$ $\left(\gamma=3, l_{\text {in }} / d=5\right)$.
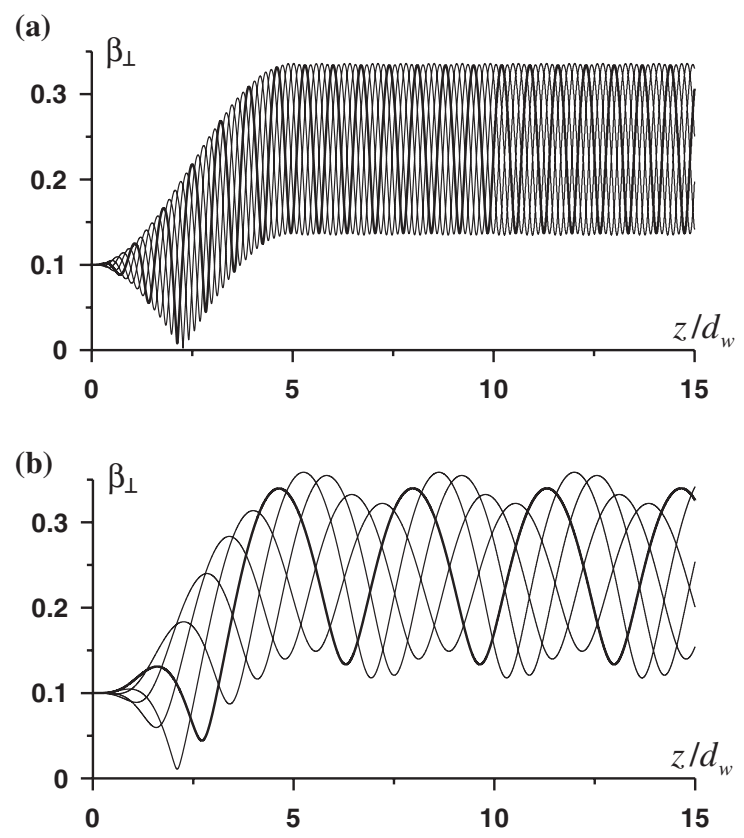

FIG. 4. Trajectory of electrons $\beta_{\perp}(z)$ having the same absolute value of initial transverse velocity but different azimuthal angles to the axis at the wiggler entrance $\left(\gamma=3, l_{i n} / d=5\right)$ : (a) $f / f_{c r}=-0.4, \alpha_{w}=1.0$ and (b) $f / f_{c r}=0.65, \alpha_{w}=0.25$. which agree well with the results of the above analytical consideration. Figure 3 shows the electron transverse velocity pumped in the wiggler $\beta_{\perp}=v_{\perp} / c=p_{\perp} / \gamma$ (where $p_{\perp}=\sqrt{p_{1}^{2}+p_{2}^{2}}$ is the dimensionless electrons transverse momentum) versus the axial coordinate $z$ for the fixed length of tapered section $l_{i n}$ and different correlation $\omega_{H} / \Omega_{b}$ (i.e. $f / f_{c r}$ ) and amplitude of the wiggler field $\alpha_{w}$, which provide the same value of the transverse velocity. At the fixed $l_{\text {in }}$ amplitude of the parasitic cyclotron oscillations is increased when the gyrofrequency approaches the cyclotron resonance value $\omega_{H}^{\text {res }} \approx \Omega_{b}$ (see Fig. 3). Obviously, to improve quality of the helical electron beam formation (i.e. decrease the value of the parasitic cyclotron oscillations) one has to shift apart from the cyclotron resonance to the high "positive" axial magnetic fields or to the "negative" axial magnetic fields in Fig. 2. The first case corresponds to a conventional guide field orientation and the second case is a so-called reversed guide field regime [6,7]. In the last regime (marked by the "-" sign here and further), the rotation of the electrons in the helical wiggler field is opposite to the cyclotron rotation in the guide field. Curve 1 in Fig. 3 corresponds to this regime, and we see that amplitude of parasitic oscillation is negligibly small. For conventional orientation to provide the same suppression of parasitic oscillation, the absolute value of the magnetic field should be in several times higher.

For a fixed ratio $\omega_{H} / \Omega_{b}$ suppression of the parasitic cyclotron oscillations can be achieved by increasing the length of the tapered wiggler section. For the guide magnetic field, which is not too close to the cyclotron resonance, a 5-7 period tapering of the wiggler is sufficient for the parasitic cyclotron oscillations to be less than $10 \%$ of the bounce oscillations $\left(\Delta \beta_{\text {cycl }} / \beta_{\perp *} \leq 0.1\right)$.

It is known that the main factor decreasing the FEL efficiency is a spread in the parameters of the driving beams. Usually in high-power microwave generators the electrons are injected from the equipotential cathode and the energy spread in such a beam is relatively small. The so-called initial position spread caused by finite width (diameter) of the beam may be prevented by using the wigglers with the improved homogeneous transverse structure [35]. Suppression of the initial velocity spread, which for the intensive relativistic electron beams can be estimated as $\Delta \beta_{\| 0} / \beta_{\| 0} \approx 1 \%-5 \%$, seems to be a more complicated problem in experimental realization of powerful millimeter generators.

Influence of the initial velocity spread on the quality of the electron beam pumped in a tapered wiggler section for different operating regimes (different ratio $\omega_{H} / \Omega_{b}$ ) is shown in Fig. 4. Different curves in Fig. 4 correspond to the trajectories of electrons having the same energies and longitudinal velocities but different azimuthal angles to the axis at the wiggler entrance. It is evident from Fig. 4(a) that in the regimes far from the cyclotron resonance these electrons in the regular wiggler section oscillate around a 
stationary helical trajectory [given by (10)] in accordance with the analytical consideration of particle motion in the phase plane described above. Particles with different initial azimuthal angles possess only different phases of the oscillations. Amplitude of these oscillations is equal to the initial parasitic transverse velocity $\beta_{\perp \text { par }}$ at the wiggler entrance [Fig. 4(a)]. It is important to emphasize that in the regular wiggler section all the particles possess approximately equal average (over the beating period $\omega_{H}-\Omega_{b}$ ) transverse and, hence, longitudinal velocities. As a result, during the interaction with the rf wave in the regular wiggler section the resonance condition (2) is practically the same [with accuracy $\beta_{\perp p a r}^{2}$, compare with formulas (36)-(38) further] for all the beam fractions and, obviously, the same conditions for energy extraction. The analysis of the e-beam/rf wave interaction described below confirms the low sensitivity to initial velocity spread in the regimes far from the cyclotron resonance.

In the regimes closer to the cyclotron resonance for a fixed length of the tapered section, the amplitude of the particle oscillations around the helical trajectory increases [Fig. 4(b)]. Simultaneously, the period of the beating of the gyro-oscillation and bounce oscillation is also increased. Both these factors result in an increase in the difference of the average value of transverse and longitudinal velocities for different electrons. This leads to different interaction conditions (2) for different beam fractions and a decrease in the efficiency of energy extraction during the rf interaction.

\section{AVERAGE APPROACH TO ELECTRON-WAVE INTERACTION}

Let us consider here the interaction between the beam electrons and the rf field of a high-Q cavity positioned within the regular wiggler section. We suppose that synchronous with the electrons component of the rf field in a cavity has a structure of a plane circularly polarized wave and can be presented by the vector potential

$$
A_{+}=A_{x}+\mathrm{i} A_{y}=A_{s}(z) \mathrm{e}^{\mathrm{i} \omega t-\mathrm{i} h z} .
$$

The motion of electrons in the wiggler field (4) and the rf wave (21) in the presence of the axial guide magnetic field can be described by the following equations:

$$
\begin{aligned}
\frac{\mathrm{d} p_{1}}{\mathrm{~d} Z} & =p_{2}\left(1-\frac{f}{p_{\|}}\right)-\left(\frac{\gamma}{p_{\|}}-1\right) \operatorname{Im}\left[\alpha_{s} \mathrm{e}^{\mathrm{i} \theta}\right], \\
\frac{\mathrm{d} p_{2}}{\mathrm{~d} Z} & =-\alpha_{w}-p_{1}\left(1-\frac{f}{p_{\|}}\right)+\left(\frac{\gamma}{p_{\|}}-1\right) \operatorname{Re}\left[\alpha_{s} \mathrm{e}^{\mathrm{i} \theta}\right], \\
\frac{\mathrm{d} \gamma}{\mathrm{d} Z} & =\frac{1}{p_{\|}} \operatorname{Re}\left[\left(\mathrm{i} p_{1}+p_{2}\right) \alpha_{s} \mathrm{e}^{\mathrm{i} \theta}\right], \\
\frac{\mathrm{d} \theta}{\mathrm{d} Z} & =g\left(\frac{\gamma}{p_{\|}}-1\right)-1,
\end{aligned}
$$

where $\theta=\omega t-h z-h_{w} z$ is the electron phase with respect to the synchronous wave, $h=\omega / c, \alpha_{s}=e h A_{s} /$ $h_{w} m c^{2}$ is the dimensionless signal amplitude, $p_{\|}=$ $\sqrt{\gamma^{2}-1-p_{1}^{2}-p_{2}^{2}}$ and $g=\omega / h_{w} c$ is the Doppler frequency up-conversion factor. Under resonance condition (2) this factor is estimated as

$$
g \approx \frac{2}{\gamma^{-2}+\beta_{\perp}^{2} / \beta_{\|}^{2}} \gg 1
$$

The FEL studies are basically concentrated on a description of regimes with high frequency up-conversion $g \approx \gamma^{2}$. Obviously, in this case the electron transverse velocity should be restricted by the condition

$$
\beta_{\perp} \leq \gamma^{-1}
$$

that leads to fulfillment of criterion (5) and justifies exploiting of the 1D model.

Boundary conditions to Eq. (22) for a monoenergetic electron beam, which is unmodulated at the entrance of the interaction space $Z=Z_{0}$, have the form

$$
\begin{aligned}
p_{1}\left(Z=Z_{0}\right) & =p_{10}, \quad p_{2}\left(Z_{0}\right)=p_{20}, \\
\gamma\left(Z_{0}\right) & =\gamma_{0}, \quad \theta\left(Z_{0}\right)=\theta_{0} \in[0,2 \pi) .
\end{aligned}
$$

The electron efficiency is given by the relation

$$
\eta=\frac{1}{2 \pi} \int_{\mathrm{Z}_{0}}^{\mathrm{Z}} \frac{\gamma_{0}-\gamma}{\gamma_{0}-1} \mathrm{~d} \theta_{0}
$$

Let us assume that at the entrance of the interaction space the electrons move along the steady-state helical trajectory (10) or near this trajectory and the signal wave amplitude is so

$$
\frac{\alpha_{s}}{\gamma_{0} \Gamma_{0}} \ll 1,
$$

that the change in electron energy during the period $2 \pi / \Omega$ is relatively small. In such an approximation it is appropriate to introduce the current (depending on the electron energy) equilibrium state $p_{\perp *}(\gamma)$ which is determined by relation (10), and represent the transverse momentum as

$$
p_{1}=p_{\perp *}(\gamma)+\rho_{1}, \quad p_{2}=\rho_{2},
$$

where $\rho_{1,2}$ describe small oscillations of the electrons around the steady-state trajectory (10).

Approximation (27) allows expansion of the right sides of Eq. (22) over $\left|\rho_{1,2}\right| \ll\left|p_{\perp *}\right|$. This approach is described in the Appendix. The analysis carried out demonstrated that the reduced equations depend on the electron phases $\theta$ and $\chi=\int_{Z_{0}}^{Z} \Gamma \mathrm{d} Z$ in synchronous wave and wiggler field. As a result, we can average these formulas in the conditions of the resonances (2) or (3) (see Appendix for details) and get the simplified equations which describe electronwave interactions under these resonances. 


\section{WIGGLER RESONANCE BETWEEN THE ELECTRONS AND THE RF WAVE}

Under the condition of the wiggler resonance (2) the average motion equation (A8) may be presented as

$$
\begin{aligned}
\frac{\mathrm{d} \gamma}{\mathrm{d} Z}= & -\frac{\alpha_{s} p_{\perp *}}{p_{\| *}} \sin \theta, \\
\frac{\mathrm{d} \theta}{\mathrm{d} Z}= & g\left(\frac{\gamma}{p_{\| *}}-1\right)-1+\frac{\alpha_{s} \gamma p_{\perp *}}{p_{\| * *}^{3} R \Gamma} \cos \theta \\
& +\frac{g \gamma}{2 p_{\| * *}^{2} p_{\perp *}} \frac{\left|C_{+0}\right|^{2} p_{\perp 0}}{p_{\| 0}}
\end{aligned}
$$

(the sign of averaging "-” is omitted here and further).

From Eq. (A10), one can see that if before entering the interaction space the electrons in the regular wiggler section move along the equilibrium helical trajectory $C_{+0}=0$ then $C_{+}(Z) \equiv 0$. It means that under the traditional wiggler resonance (2) during the process of the interaction with the signal wave electrons stay in equilibrium trajectory; however parameters of this trajectory are altered in accordance with the change in the electron energy $\gamma$. As a result, for the electrons moving along the steady-state trajectory $\left(C_{+} \equiv 0\right)$ the averaged equations of the electron motion are reduced to two equations (28), which may be presented in a canonical form:

$$
\frac{\mathrm{d} \gamma}{\mathrm{d} Z}=-\frac{\partial \mathcal{H}}{\partial \theta}, \quad \frac{\mathrm{d} \theta}{\mathrm{d} Z}=\frac{\partial \mathcal{H}}{\partial \gamma}
$$

with the Hamiltonian

$$
\begin{aligned}
\mathcal{H} & =\int_{\gamma_{0}}^{\gamma}\left[g\left(\frac{\gamma}{p_{\| *}}-1\right)-1\right] d \gamma-\frac{\alpha_{s} p_{\perp *}}{p_{\| *}} \cos \theta \\
& =g\left[p_{\| *}-\gamma+p_{\perp *}\left(\frac{1}{p_{\| *}}-\frac{1}{2 f}\right)\right]-\gamma-\frac{\alpha_{s} p_{\perp *}}{p_{\| *}} \cos \theta .
\end{aligned}
$$

The dependencies of $p_{\perp *}(\gamma), p_{\| * *}(\gamma)$, and $\Gamma(\gamma)$ in Eqs. (28) and (29) are assigned implicitly through the relations (10) and (14). When gyrofrequency and bounce frequency are sufficiently different and when the transverse momentum of the electrons in the wiggler is small compared to the axial momentum [i.e. condition (16) is satisfied], these dependencies may be presented in the explicit form:

$p_{\perp *} \approx-\frac{\alpha_{w}}{1-\omega_{H 0} / \gamma \Omega_{b}}, \quad p_{\| *} \approx \sqrt{\gamma^{2}-1}, \quad \Gamma \approx 1-\frac{\omega_{H 0}}{\gamma \Omega_{b}}$.

Under these simplification Eq. (28) can be reduced to the form

$$
\begin{aligned}
\frac{\mathrm{d} \gamma}{\mathrm{d} Z} & =\frac{\alpha_{s} \alpha_{w}}{\sqrt{\gamma^{2}-1}\left(1-\omega_{H 0} / \gamma \Omega_{b}\right)} \sin \theta, \\
\frac{\mathrm{d} \theta}{\mathrm{d} Z} & =-g-1+\frac{g \gamma}{\sqrt{\gamma^{2}-1}}+\frac{g \gamma \alpha_{w}^{2}-2 \alpha_{s} \alpha_{w} \gamma \cos \theta}{2\left(\gamma^{2}-1\right)^{3 / 2}\left(1-\omega_{H 0} / \gamma \Omega_{b}\right)} .
\end{aligned}
$$

In this form the motion equations for a FEL with a guide magnetic field can be obtained within the framework of the averaged approach based on the ponderomotive force for magnetically guided electrons in the wiggler and rf fields [29].

\section{A. Small-signal approximation}

In the small-signal regime $\alpha_{s} \rightarrow 0$ the equations (28) for the electrons moving along the steady-state trajectory $\left(C_{+} \equiv 0\right)$ may be solved using the method of consecutive approximations. Following this method in the first approximation (which does not vanish under averaging over the initial electron phases and proportional to $\alpha_{s}^{2}$ ), we get the following expression for the efficiency of the small-signal regime:

$$
\begin{aligned}
\eta_{\text {s.s. }} & =\frac{\alpha_{s}^{2} L^{2}}{1-\gamma_{0}^{-1}} \Phi, \\
\Phi & =\left[L g \mu_{0} \frac{p_{\perp 0}^{2}}{8 \gamma_{0}^{2} p_{\| 0}^{2}} \frac{\mathrm{d}}{\mathrm{d} \Psi}\left(\frac{\sin \Psi}{\Psi}\right)^{2}-\frac{\alpha_{w} p_{\perp 0}}{2 p_{\| 0}^{4} \Gamma_{0}^{2}}\left(\frac{\sin \Psi}{\Psi}\right)^{2}\right],
\end{aligned}
$$

where $\Psi=\Delta L$ is the transient angle, $\Delta=g\left(\gamma_{0} / p_{\| 0}-1\right)-1$ is the initial mismatch from the wiggler resonance, $L=h_{w} l, l$ is the length of the interaction space.

For a FEL with high-Q resonator the power balance condition in a stationary regime of oscillations is valid:

$$
\frac{\omega W}{Q}=I \eta \frac{m c^{2}}{e}\left(\gamma_{0}-1\right)
$$

where $W$ is the energy stored in the resonator of the quality $Q$. In the small-signal regime substituting expression (32) in Eq. (33) we find the starting current of the oscillations,

$$
I_{\text {start }}=\frac{m c^{3}}{e} \frac{S}{2 \gamma_{0} \lambda l Q \Phi} .
$$

Note that to get Eq. (34) we supposed the rf wave inside the resonator of length $l$ and the cross section $S$ as a standing wave having transverse homogeneous profile. It is important to note that expression (32) is identical to the one obtained in [28] in linear approximation directly from Eq. (22) under the assumption of the wiggler resonance (2).

The first term in the expression for $\Phi$ in (32) describes the e-beam/rf-wave energy exchange under the inertial bunching caused by the dependence of the longitudinal velocity of electrons $v_{\| * *}=c \beta_{\| * *}$ on their energy $\gamma$. This term is proportional to the inertial bunching parameter [2,21],

$$
\mu=-\gamma \frac{\mathrm{d} \beta_{\| * *}^{-1}}{\mathrm{~d} \gamma}=-\gamma \frac{\mathrm{d}}{\mathrm{d} \gamma} \frac{\gamma}{p_{\| *}}=\frac{\gamma \alpha_{w}\left(\alpha_{w}-p_{\perp *}^{3}\right)}{p_{\perp *}^{2} p_{\| * *}^{3} \Gamma^{2}} .
$$

This parameter is inversely proportional to the effective longitudinal mass of the electron oscillators $M_{\|}=$ $d E / d p_{\| *}=m c^{2} / \mu \beta_{\| *}^{2}$. Note that in [22-27] a similar parameter is called the magnetic coupling parameter. 
The second term for parameter $\Phi$ in (32) is responsible for an e-beam/rf-wave interaction caused by a so-called force bunching. This bunching mechanism associated with the dependencies of $p_{\perp *}(\gamma), p_{\| * *}(\gamma)$ in Eq. (28a) for electrons energy and the term proportional to $\alpha_{s}$ in Eq. (28b) for electrons phase. Note that both mechanisms give equal contribution to the second term in the expression for $\Phi$ in Eq. (32). The force bunching leads to the absorption of energy by the electrons from the rf wave for a group of stationary orbits II (where $p_{\perp 0}>0$ ) and conversely to the generation of radiation for the group I orbits (where $p_{\perp 0}<0$ ). Within the framework of the averaged equations (28) this is explained by the fact that for the stationary orbits I during the rf interaction the pitch factor $p_{\perp *} / p_{\| * *}$ of the decelerating electrons increased and they start to interact with the rf wave more effective than the accelerating electrons. As a result, on average over the initial phase $\theta_{0}$ deceleration prevails over acceleration. With the stationary orbits II the situation is just opposite, i.e., the pitch factor for decelerated electrons decreases and the particles acceleration and electromagnetic energy absorption prevail.

Let us now include in the consideration the electrons, which possess small oscillations around the steady-state helical trajectory. As it was shown in Sec. II, the electrons undergo these oscillations in the regular wiggler section if the adiabaticity of the wiggler entrance is violated or the electrons possess the initial cyclotron oscillations at the wiggler entrance. In this case the boundary conditions at the beginning of the interaction space have the form (A5c), i.e. $C_{+}\left(Z=Z_{0}\right)=C_{+0} \neq 0$. For these electrons in a small-signal regime the last term in the right side of Eq. (28) contributes in the effective mismatch from the wiggler resonance

$$
\Delta_{\text {eff }}=g\left(\frac{\gamma_{0}}{p_{\| 0}}-1\right)-1+\frac{g \gamma_{0}}{2 p_{\| 0}^{3}}\left|C_{+0}\right|^{2}
$$

and also results in appearance of an additional term in the expression for the efficiency of the small-signal regime (32):

$$
\begin{aligned}
\eta_{\text {s.s. }}= & \frac{\alpha_{s}^{2} L^{2}}{1-\gamma_{0}^{-1}}\left\{\Phi+\frac{g p_{\perp 0}^{2}\left|C_{+0}\right|^{2}}{16 \gamma_{0} p_{\| 0}^{7}}\left[1+\gamma_{0}^{2}\left(1+\frac{f p_{\perp 0}}{\alpha_{w} p_{\| 0}}\right)\right]\right. \\
& \left.\times \frac{\mathrm{d}}{\mathrm{d} \Psi}\left(\frac{\sin \Psi}{\Psi}\right)^{2}\right\}
\end{aligned}
$$

Thus, this term makes a contribution in the process of inertial bunching of the electrons. Comparing (37) with (32) and (35) we see that the effective bunching parameter $\mu_{\text {eff }}$ for the electrons oscillating around the steady-state helical trajectory equals

$$
\mu_{\mathrm{eff}}=\mu+\frac{\gamma\left|C_{+0}\right|^{2}}{2 p_{\| * *}^{5}}\left[1+\gamma^{2}\left(1+\frac{f p_{\perp *}}{\alpha_{w} p_{\| * *}}\right)\right] .
$$

Thus, according to (28) and (36)-(38) the initial parasitic transverse oscillations $C_{+0}$ present in the beam at the entrance of the interaction space results in the appearance of additional terms proportional only to $\left|C_{+0}\right|^{2}$ in the motion equations. This leads to a weak dependence of efficiency on the velocity spread (i.e. spread over the value $C_{+0}$ ) for an FEL with a guide magnetic field. The computer simulations of the nonlinear stage of e-beam/rf-wave interaction presented below confirm this conclusion.

\section{B. Nonlinear stage of e-beam/rf-wave interaction}

To analyze the nonlinear stage of interaction between the electrons and the rf wave several specific regimes of FEL operation have to be separated.

\section{Regimes far from the cyclotron resonance including a reversed guide field regime}

When the gyrofrequency of the electrons differs greatly from the cyclotron resonance with bounce oscillations, i.e., in regimes with a strong guide field at the stationary orbits II or with weak and reversed (marked by sign "-") guide field at the group I orbits, the dependencies $p_{\perp *}(\gamma), p_{\| *}(\gamma)$, $\mu(\gamma)$, etc. become very weak (compare with Figs. 2 and 5). This results in a small change of these values during the rf interaction and allows in nonlinear motion equations (28) to be replaced by its initial values, i.e. $p_{\perp, \| *}=p_{\perp, \| 0}$. Moreover, if the interaction length is high enough,

$$
L \mu g \Gamma \gg 1
$$

and the rf-field amplitude is not too large (26) then the inertial bunching becomes dominant and the second term in (32) becomes negligible. Thus, in these regimes we can also neglect the term proportional to $\alpha_{s}$ in the equation for the electron phases (28b). Simultaneously, under condition (39) the changes in the electron energies are relatively small [compare with relation (43) below]. In these assumptions Eq. (28) can be simplified and presented in a pendulumlike form (compare with [2]):

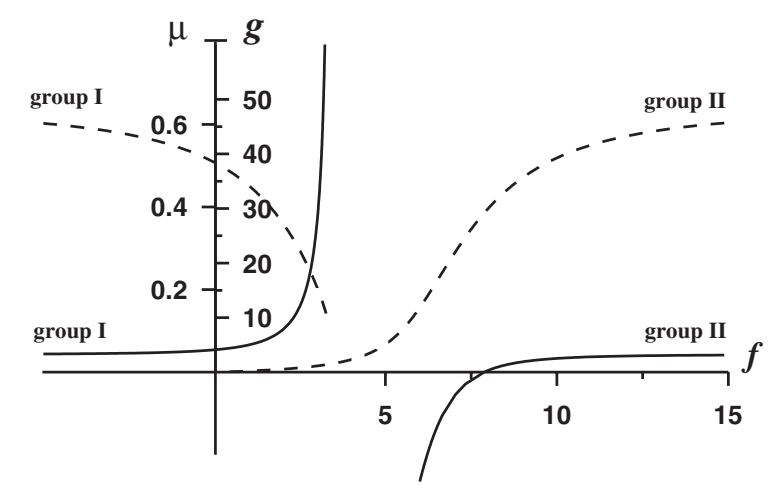

FIG. 5. The bunching parameter $\mu$ (solid line) and frequency up-conversion coefficient $g$ (dashed line) at the equilibrium helical trajectory for the groups of orbits I and II as a function of guide magnetic field strength $f\left(\gamma=5, \alpha_{w}=0.1\right)$. 


$$
\frac{\mathrm{d} w}{\mathrm{~d} Z}=\frac{\alpha_{s} p_{\perp 0}}{\gamma_{0} p_{\| 0}} \sin \theta, \quad \frac{\mathrm{d} \theta}{\mathrm{d} Z}=\Delta_{\mathrm{eff}}+g \mu_{\mathrm{eff}} w,
$$

where $w=\left(\gamma_{0}-\gamma\right) / \gamma_{0}$ is relative variation of the electron energy. Introducing the new variables

$$
\begin{gathered}
\xi=\left(Z-Z_{0}\right) \sqrt{\frac{\alpha_{s} \mu_{\mathrm{eff}} g p_{\perp 0}}{\gamma_{0} p_{\| 0}}}, \quad u=w \sqrt{\frac{g \mu_{\mathrm{eff}} \gamma_{0} p_{\| 0}}{\alpha_{s} p_{\perp 0}}}, \\
\bar{\Delta}=\Delta_{\mathrm{eff}} \sqrt{\frac{\gamma_{0} p_{\| 0}}{\alpha_{s} \mu_{\mathrm{eff}} g p_{\perp 0}}},
\end{gathered}
$$

it is easy to minimize the number of independent parameters and reduce (40) as

$$
\frac{\mathrm{d} u}{\mathrm{~d} \xi}=\sin \theta, \quad \frac{\mathrm{d} \theta}{\mathrm{d} \xi}=\hat{\Delta}+u \operatorname{sign} \mu .
$$

The efficiency in the new variables is determined by the relations

$$
\eta=\bar{\eta} \sqrt{\frac{\alpha_{s} p_{\perp 0}}{g \mu_{\mathrm{eff}} \gamma_{0} p_{\| 0}}}, \quad \eta=\frac{1}{2 \pi} \int_{0}^{2 \pi} u \mathrm{~d} \theta_{0} .
$$

The maximum of the normalized efficiency $\hat{\eta} \approx 1.37$ is reached at $\Delta_{\mathrm{opt}} \approx-1.3 \operatorname{sign} \mu$ and $\xi_{\mathrm{opt}} \approx 3.9$. According to (42), the total efficiency at constant amplitude of the rf wave and pitch angle of the electrons $p_{\perp 0} / p_{\| 0}$ is determined by the bunching parameter $\mu_{\text {eff }}$. In the regimes, when the guide magnetic field is far from the cyclotron resonance value $f_{c r} \approx \gamma$ (i.e. $\omega_{H} \approx \Omega_{b}$ ), for the electrons moving along the helical equilibrium trajectory the bunching parameter is given by $\mu \approx \gamma^{-2}$ (Fig. 5). In this regime under the high Doppler up-conversion $g \approx \gamma^{2} \gg 1$ the electron efficiency is defined by the number of bounce oscillations $N$ (i.e. number of the wiggler periods) in the interaction length [2],

$$
\eta \approx \frac{1}{\mu g N} \approx \frac{1}{N}
$$

Computer simulations of the e-beam/rf-wave interaction using the nonlinear equations (A8) as well as using full (nonaverage) equations (22) confirm results of the above analysis. Figure 6 presents results of the computer simulations for an "ideal" (i.e. without any spread of parameters) electron beam moving along the helical equilibrium trajectory and interacting with the rf wave of constant amplitude within the interaction region in the regime of a reversed guide magnetic field. (Note that in all further considerations the point " 0 " corresponds to the beginning of the rf interaction, i.e. $Z=Z_{0}$.) These simulations demonstrate that for a mildly relativistic electron beam $\gamma_{0} \approx 3$ relatively high efficiency $\eta \approx 15 \%-20 \%$ can be achieved at the interaction (cavity) length corresponding to the number of the bounce oscillations $N \approx 5-7$ for the pitch factor of electrons $p_{\perp *} / p_{\| *} \sim \gamma_{0}^{-1} \approx 0.3$, which coincides well to the estimation given by (43). Note also that the

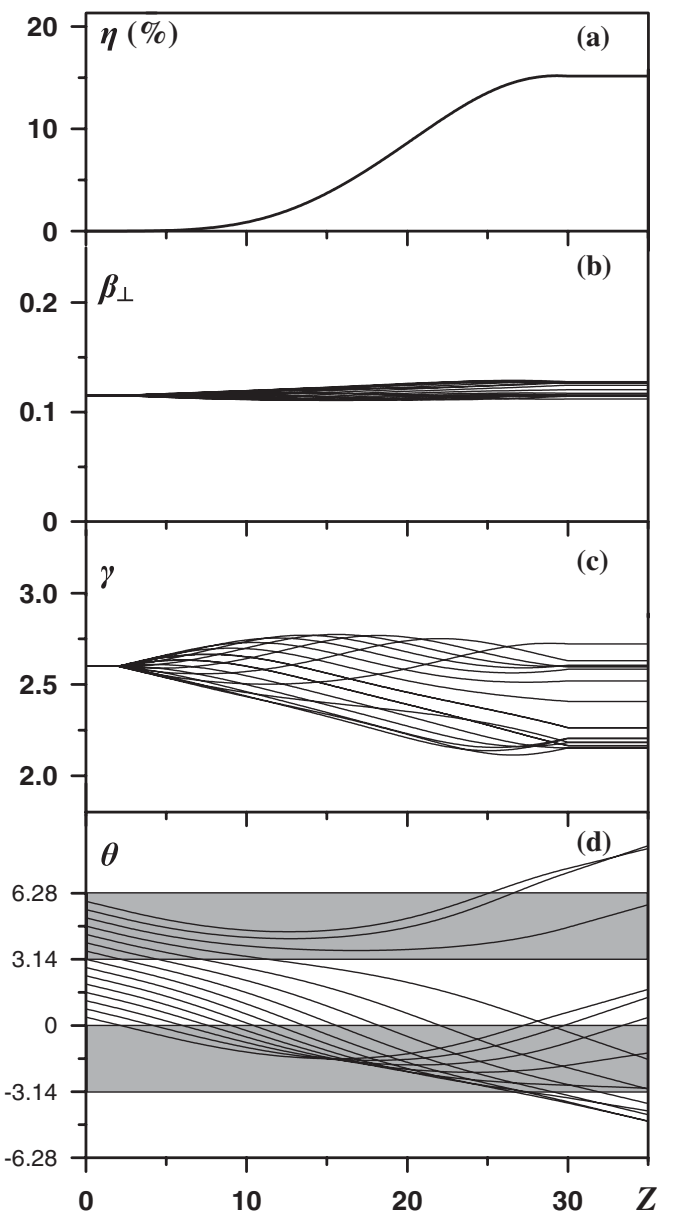

FIG. 6. FEL with a reversed guide field driven by ideal electron beam. Dependence on longitudinal coordinate of (a) electron efficiency as well as (b) transverse velocity, (c) energy, and (d) phase with respect to the rf wave for electrons with different initial phases (the hatched zones represent decelerated phases): $\gamma_{0}=2.6, \alpha_{w}=0.5, \alpha_{s}=0.15, f=-1.2$, $\mu_{0}=0.22, g=6.5, \Delta=-0.16$.

normalized radius of electron oscillations $R_{b}=h_{w} r_{b}$ in this case amounts to $R_{b} \sim 0.04-0.05$ that in accordance with (5) and (23) corroborate validity of the model used.

An advantage of the FEL operation in the regimes far from the cyclotron resonance is the low sensitivity to the initial velocity spread in the beam (compare with [36,37]). In Sec. II it was shown that in these regimes the electrons, which possess at the wiggler entrance the same energies and longitudinal velocities but different azimuthal angles to the axis, inside the interaction region oscillate around the equilibrium helical trajectory with the same amplitude, which is approximately equal to their initial parasitic transverse velocity $\beta_{\perp \text { par }}$ [see Fig. 4(a)]. In the framework of Eq. (28) all these particles possess the same value $\left|C_{+0}\right|=\gamma_{0} \beta_{\perp \text { par }}$ and, therefore, identical energy extraction during the $\mathrm{rf}$ interaction. Therefore, to analyze an electron beam with velocity spread it is sufficient to consider only those beam fractions which have different values 


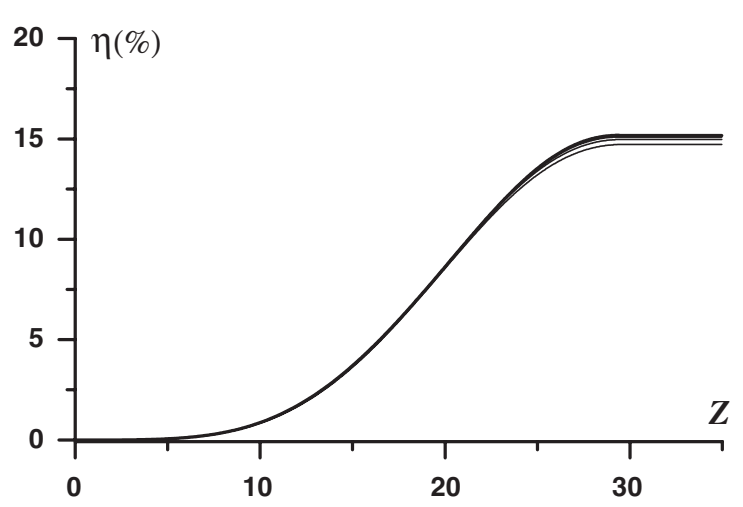

FIG. 7. FEL with a reversed guide field. Dependence of electron efficiency on longitudinal coordinate (for parameters given in Fig. 6) for the beam fractions with different initial parasitic transverse velocity at the entrance of the interaction space. The maximum initial parasitic transverse velocity equals $50 \%$ of the operating transverse velocity.

of the initial parasitic transverse velocity $\beta_{\perp \text { par }}$ and to exclude the fractions which have different phases of the initial transverse velocity. Figure 7 presents dependencies of the electron efficiency for the beam fractions having different values of the initial transverse velocity $\beta_{\perp \text { par }}$ at the entrance of the interaction space [i.e. values $C_{+0}$ in the Eq. (28)]. Computer simulation shows that even for an initial parasitic velocity which is equal to half of the operating velocity, i.e. $\left|C_{+0}\right| / p_{\perp 0}=50 \%$ (under the parameters given in Fig. 7 this corresponds to the initial longitudinal velocity spread $\Delta \beta_{\| 0} / \beta_{\| 0} \approx 5 \%$ ), the electron efficiency is approximately the same for all the beam fractions. The total FEL efficiency averaged over all the fractions (in the simulations we assume equal numbers of particles with different initial parasitic velocities from 0 to $\Delta \beta_{\perp \text { par }}$ at the wiggler entrance) versus the value of the initial velocity spread $\Delta \beta_{\perp \mathrm{par}} / \beta_{\perp *}$ is given in Fig. 8

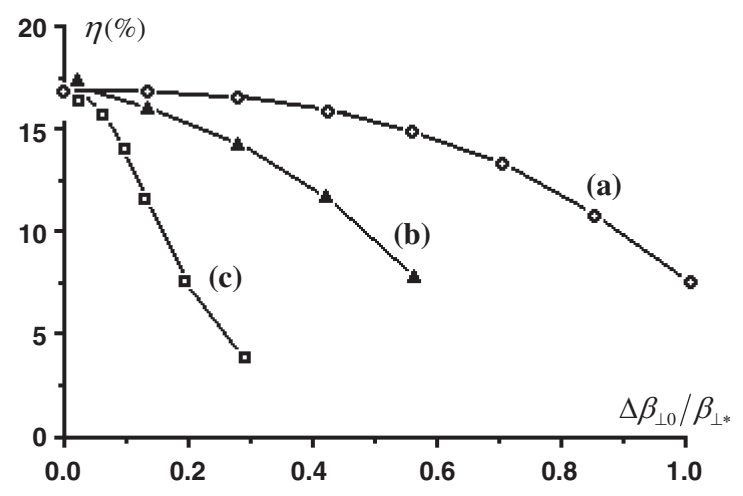

FIG. 8. Influence of initial velocity spread. The electron efficiency averaged over all the beam fractions versus the initial parasitic transverse velocity presented in the beam: (a) FEL with reversed guide field for parameters given in Fig. 6; (b) FEL with conventional guide field for parameters given in Fig. 10; (c) CARM of the same frequency, electron energy, and pitch factor. (curve "a"). Even for an electron beam with a rather large initial parasitic transverse velocity $\Delta \beta_{\perp \text { par }} / \beta_{\perp *} \sim 50 \%$ a decline of FEL efficiency averaged over all the fractions does not exceed $\sim 1 \%-2 \%$ of the efficiency of a FEL driven by an ideal electron beam.

It is appropriate here to compare the sensitivity to velocity spread for the different well-known version of the relativistic electron maser like cyclotron autoresonance maser (CARM). In this device for electron beam formation, a scheme similar to a FEL is used: rectilinear (with the accuracy of the initial velocity spread) electron beam guided by an axial magnetic field is generated by a relativistic gun and then the operating transverse velocity of the particles is imparted by a relatively short (nonadiabatical) pumping systems (kickers) and remained constant after that in a uniform axial magnetic field [38,39]. Influence of initial velocity spread on the quality of helical electron beam formation for CARMs was studied in [38]. It was shown that in the kickers electrons having initially the same energies and longitudinal velocities but different azimuthal angles to the axis at the entrance get different transverse and, hence, longitudinal velocities when passing through the pumping system. The maximum difference in the transverse velocities for different fractions is equal to the double initial parasitic transverse velocity $2 \beta_{\perp \text { par }}$, in contrast with FEL operating in the regimes far from the cyclotron resonance, where the difference in the average transverse velocity for different fractions is in the order of $\beta_{\perp \text { par }}^{2}$ [see Fig. 4(a) and compare with Eqs. (28) and (36)-(38)]. A big difference (in comparison with the FEL) in velocities of different fractions results in violation of the rf-resonance conditions producing a significant reduction in the efficiency of CARMs (see [36] for details).

In accordance with the above consideration the computer simulations of the e-beam/rf-wave interaction [36] show that for a CARM (Fig. 8, curve "c") an initial parasitic transverse velocity equals to $20 \%$ of the operating transverse velocity leads to a decrease of efficiency by a factor of 2. Note that the parameters for the FEL and the CARM in the simulations given in Fig. 8 were chosen to be same in frequencies as well as in energies and pitch angles of the electrons. Therefore, computer simulations demonstrate a potentially higher ability of the FEL with a guide magnetic field in comparison with CARMs to achieve a higher efficiency and, therefore, a higher microwave power when driven by electron beams with "real" dispersion of parameters.

\section{Regimes of weak guide field $\left(\omega_{H}<\Omega_{b}\right)$ of a conventional orientation}

In such regimes the axial guide magnetic field is closer to its resonant value (i.e. the gyrofrequency is close to the bounce frequency) compared with the reversed guide field regimes. As a result, in the tapered wiggler section of fixed length the amplitude of the electron oscillations around 
the stationary helical trajectory in the regular wiggler section as well as the spatial period of this oscillation (length of cyclotron-bounce beating) increases (compare with Figs. 3 and 4). As it follows from relation (38) the inertial bunching parameter for the electrons oscillating around the steady-state helical trajectory (for which $C_{+0} \neq 0$ ) is higher as compared with the electrons moving along this trajectory (for which $C_{+0}=0$ ). According to Eqs. (41) and (42), efficiency of energy extraction from oscillating around the helical trajectory electrons is lower. Therefore, the cyclotron oscillations in the driving beam, which are excited and increased in the regimes close to the cyclotron resonance (when the condition of the adiabaticity of the wiggler entrance is violated), result in a decrease in the FEL efficiency. Note that the optimal mismatch from the wiggler resonance decreases in this case [compare with (36)].

As discussed before, a specific feature of the regimes of weak guide field with a conventional orientation, i.e., for the group of stationary orbits I, is the increase in the transverse velocity of the electrons during the process of the rf interaction. The dependence of the transverse velocity on electron energy becomes very strong when the gyrofrequency approaches the bounce frequency. The strong change in the transverse velocity of the electrons near the cyclotron resonance is an additional reason for violation of the synchronism conditions during rf interaction. This fact is illustrated by Fig. 9 where the arrows show the change in the transverse velocity for decelerated particles in different regimes. In the regimes of weak conventional field close to the resonance value a decrease in electrons energy is accompanied by a large increase in their transverse velocity. As a result, the decelerated particles may fall on the wall of the microwave cavity, which will lead to beam current losses during the rf interaction (compare with [26]).

Computer simulations by Eq. (28) confirm that in this region of parameters during the $\mathrm{rf}$ interaction decelerated electrons experience an increase in their transverse

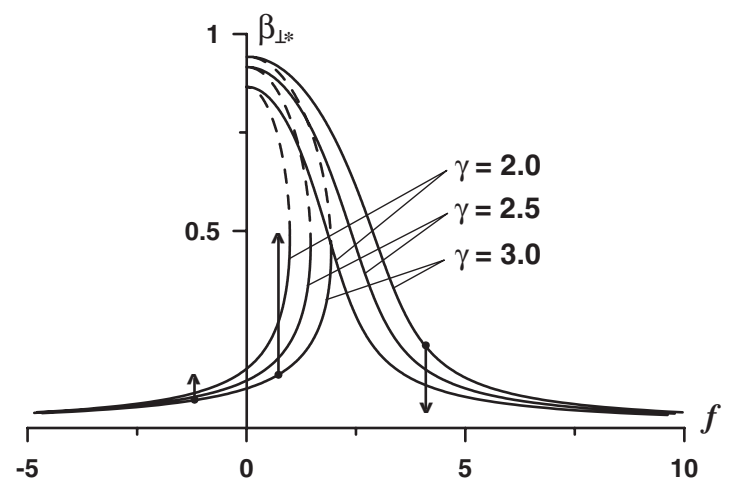

FIG. 9. The transverse velocity at the equilibrium helical trajectory for the electrons of different energies versus guide field. The arrows illustrate a change of transverse velocity for decelerated particles in different regimes.

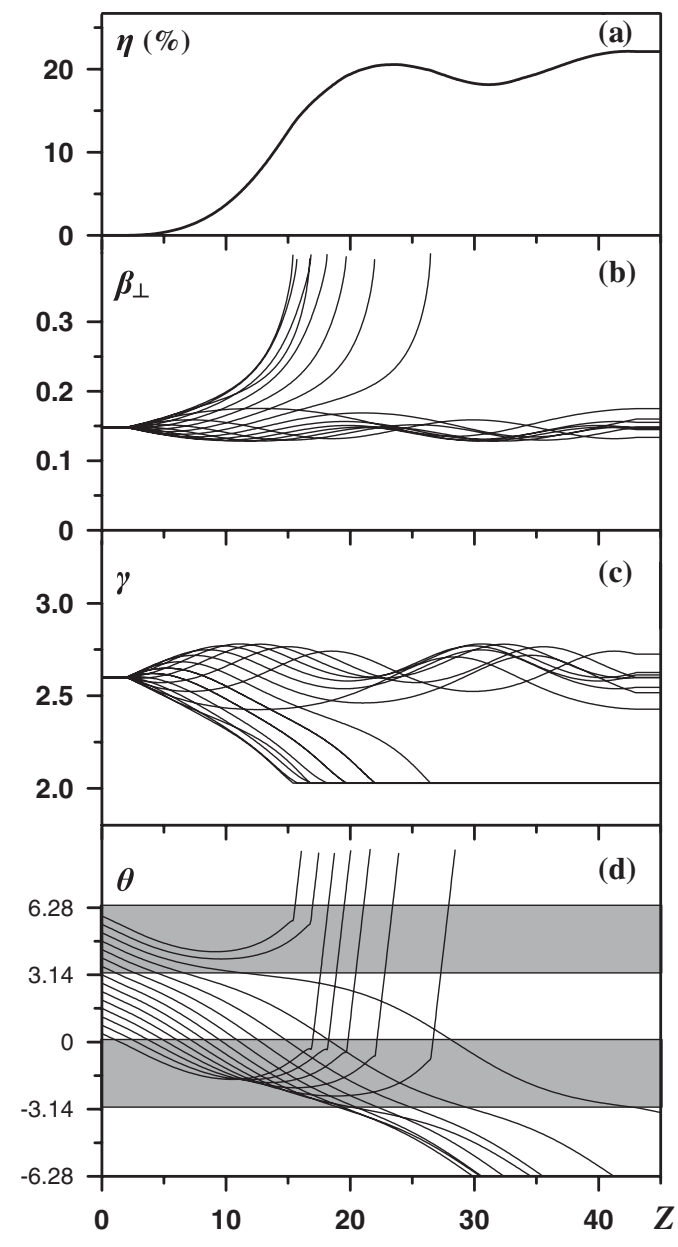

FIG. 10. FEL with a weak conventional guide field driven by an ideal electron beam. Dependence on longitudinal coordinate of (a) electron efficiency as well as (b) transverse velocity, (c) energy, and (d) phase with respect to the rf wave for electrons with different initial phases: $\gamma_{0}=2.6, \alpha_{w}=0.15, \alpha_{s}=0.15$, $f=1.2, \mu_{0}=0.25, g=6.5, \Delta=-0.28$.

velocities [Fig. 10(b)]. Transverse velocity of the spinning electrons grows up very fast and such electrons interrupt their synchronous interaction with the wave. For this reason the $1 \mathrm{D}$ trajectory model can be also effectively used for description of these regimes. The direct 3D simulations carried out in [37] corroborate this conclusion.

It is important that the process of rf interaction becomes more sensitive to the initial velocity spread (Fig. 11). For the regime simulated in Figs. 10 and 11, the presence of parasitic initial velocity in the beam of $15 \%$ of the operating transverse velocity results in a decrease of the efficiency by a factor of about 1.5. Note that in some other regimes with a conventional guide field an even higher drop of FEL efficiency was observed. In particular, decrease in efficiency is much more dramatic when the inhomogeneities of the wiggler and the rf field were taken into account [37]. Therefore, an FEL operated in regimes with weak conventional guide field is more sensitive to the initial velocity spread (see Fig. 8, curve "b") as compared with the regimes 


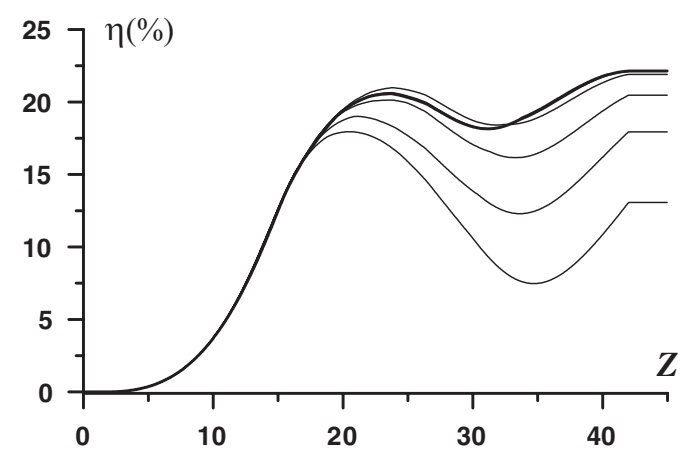

FIG. 11. FEL with a weak conventional guide field. Dependence of electron efficiency on longitudinal coordinate (for parameters given in Fig. 10) for the beam fractions with different initial parasitic transverse velocity at the entrance of the interaction space. The maximum initial parasitic transverse velocity equals $15 \%$ of the operating transverse velocity.

far from the cyclotron resonance including a reversed guide field regime. Note also that the optimal interaction length in this region of parameters also increases.

We should emphasize that regimes of weak guide field with a conventional orientation are characterized by strong dependence of initial conditions. The parameters of the interaction can be chosen so that the electrons, which increase their transverse velocities, would get to the wall of the system and, therefore, would be excluded from the energy exchange just before they would come into accelerating phase. A similar effect when the drop of beam current is accompanied by the increase of electron efficiency was described in [26] based on the full (nonaverage) equations.

In conclusion, let us note that the parameters in the simulations used above were chosen to be close to the parameters of the FEL experiments carried out in collaboration between JINR (Dubna, Russia) and IAP RAS (Nizhny Novgorod, Russia) based on linac LIU-3000 $(0.8 \mathrm{MeV} / 200 \mathrm{~A} / 200 \mathrm{~ns})$. In these experiments (see $[17,18]$ for details) the operating transverse velocity $\beta_{\perp} \approx$ 0.2 was pumped in a helical wiggler of $6 \mathrm{~cm}$ period. A selective feedback at the frequency about $30 \mathrm{GHz}$ was realized by use of Bragg resonators (a two-mirror Bragg resonator [37] either a Bragg resonator with a step of phase of corrugation [17]). For the conditions of the experiments the dimensionless parameters, for example, $\alpha_{w}=0.5$ and $f=-1.0$ correspond to $H_{w}=1 \mathrm{kOe}$ and $H_{0}=-2 \mathrm{kOe}$, respectively.

The results of theoretical consideration presented in Sec. IVA and IV B coincide well with the results of the experiments. In particular, maximum efficiency of $\sim 20 \%-30 \%$ was obtained in under the reversed guide field orientation $[17,18]$. The efficiency achieved in this experiment is a record for millimeter-wave FEL oscillators $[2,8-12]$. We have to note that these values are even slightly higher than those calculated in Fig. 6 for parameters close to the experimental values. This can be explained by the longitudinal rf-field structure in the Bragg resonators (especially in a resonator with step of phase of corrugation) being more optimal for the extraction of energy from the electron beam as compared to a constant rf-field profile inside the interaction region which was used in the simulations. Decrease in the FEL efficiency was observed in conventional guide field regimes with the strength less than the cyclotron resonance value (unfortunately, the conditions of the discussed experiments did not allow the FEL to operate with a guide field over the resonance value). It is important to note that in the regimes close to the cyclotron resonance the process of $\mathrm{rf}$ generation was accompanied with a remarkable loss in beam current [37] caused by electrons which hit the wall of the transport channel when interacting with the rf wave.

\section{Regimes of strong guide field $\left(\omega_{H} \geq \Omega_{b}\right)$ of conventional orientation}

The attractive feature of the FEL with a guide magnetic field is the possibility of effectively varying the inertial bunching parameter, i.e., the effective longitudinal mass of the electron oscillators (see Fig. 5). In particular, at the group II stationary orbits when the gyrofrequency slightly exceeds the bounce frequency, i.e. $f \approx \gamma_{0}\left(1+\alpha_{w}^{2 / 3}\right)$, the value of the bunching parameter tends to zero $\mu \rightarrow 0$ (effective mass tends to infinity). According to (43) this fact would increase FEL efficiency [21,29].

However, in high-efficiency regimes the condition of inertial bunching domination [Eq. (39)] is violated and the full (nonlinear) averaged equations of motion (28) have to be used. In analysis of these equations the dependence of the bunching parameter on the electron energy (see Fig. 12) becomes important [29,40]. If the parameters of the system are chosen so that the point $\mu_{0}$ in Fig. 12 corresponds to the initial electron energy $\gamma_{0}$, then it is evident from this diagram that the bunching parameter for decelerated electrons would decrease in absolute value

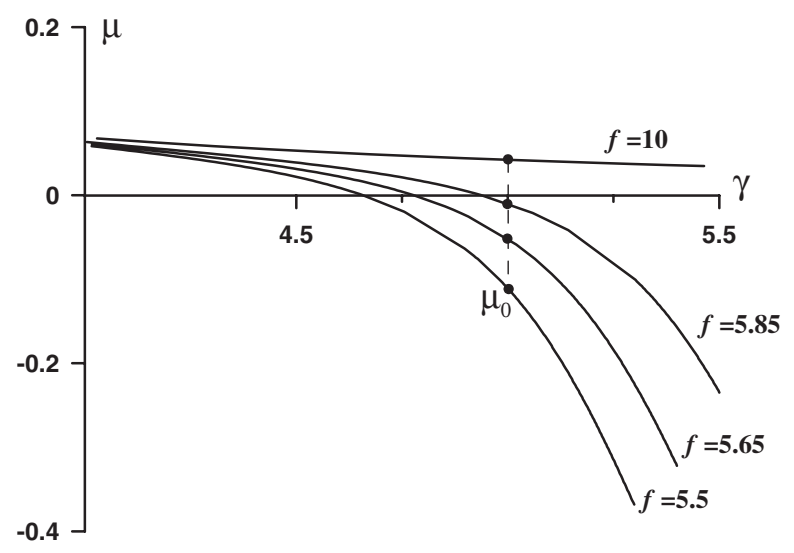

FIG. 12. The bunching parameter $\mu$ as a function of electron energy $\gamma$ at $\alpha_{w}=0.1$ and different values of guide magnetic field $f$ : (1) $f=10$; (2) $f=5.85$; (3) $f=5.65$; (4) $f=5.5$. 


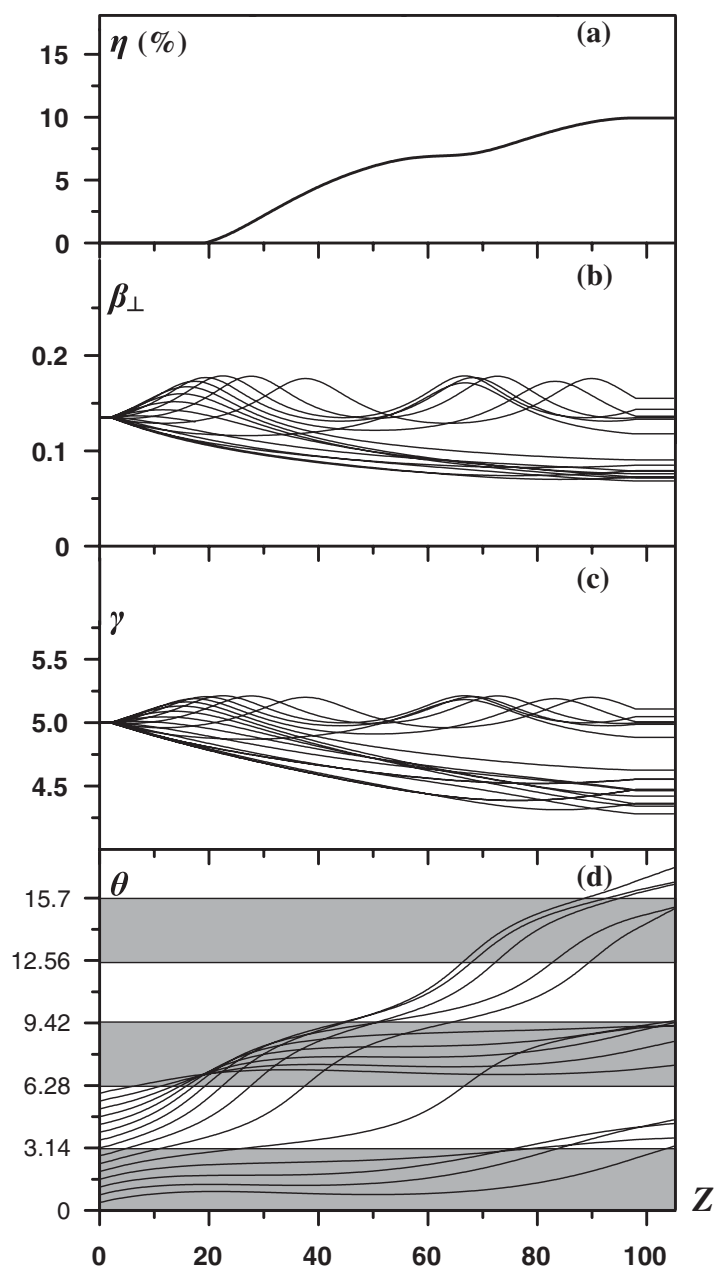

FIG. 13. FEL with a strong conventional guide field driven by an ideal electron beam. Dependence on longitudinal coordinate of (a) electron efficiency as well as (b) transverse velocity, (c) energy, and (d) phase with respect to the rf wave for electrons with different initial phases: $\gamma_{0}=5.0, \alpha_{w}=0.1, \alpha_{s}=0.05$, $f=5.6, \mu_{0}=-0.06, g=36, \Delta=0.05$.

while that for accelerating particles would increase. The bunching parameter [see (35)] characterizes the magnitude of the change in the axial velocity of the electrons as the electron energy changes. Therefore, the decelerated particles would stay in synchronism and extract the energy to the rf wave for a longer time as compared to the accelerated particles, which would absorb the rf-wave energy. In Fig. 13 we can clearly see that the particles are divided into two fractions. The first fraction possesses a small parameter $\mu$ and is kept in a decelerating phase of the rf wave for a long time while the energy of particles of the second (accelerating) fraction, whose parameter $\mu$ is large, quickly oscillates. Note that an FEL operated in the regime described above is also more sensitive to the initial velocity spread (Fig. 14).

The described method of selective discrimination of accelerated electrons results in the increase of the electron efficiency as compared with regimes far from the cyclotron

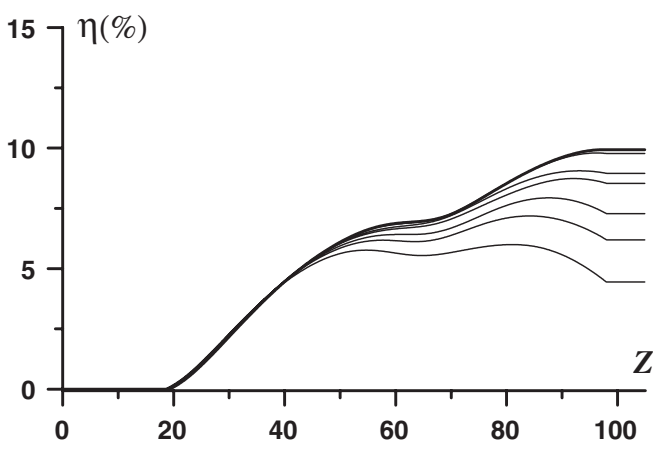

FIG. 14. FEL with a strong conventional guide field. Dependence of electron efficiency on longitudinal coordinate (for parameters given in Fig. 13) for the beam fractions with different initial parasitic transverse velocity at the entrance of the interaction space. The maximum initial parasitic transverse velocity equals $15 \%$ of the operating transverse velocity.

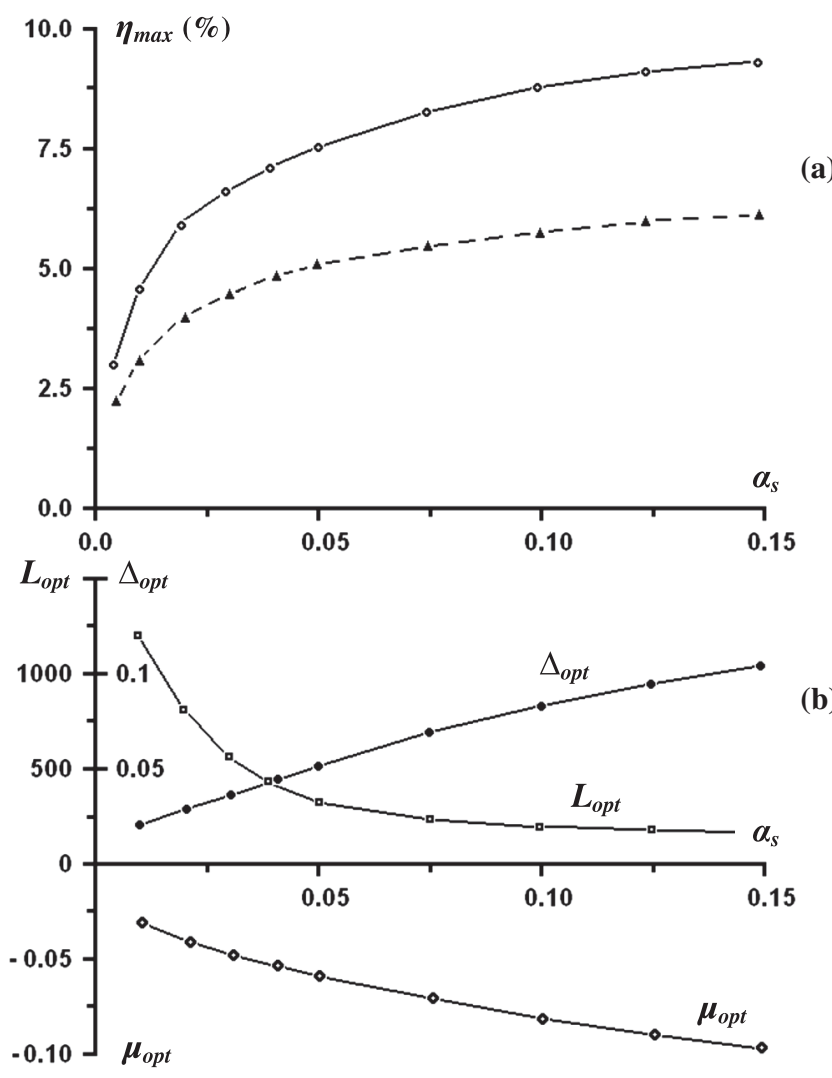

FIG. 15. The dependence of (a) maximum efficiency $\eta_{\max }$ as well as (b) optimal value of parameters $\mu_{0}^{\mathrm{opt}}, \Delta^{\mathrm{opt}}$, and $L^{\mathrm{opt}}$ on the amplitude of the signal wave $\alpha_{s}\left(\gamma_{0}=5, \alpha_{w}=0.1\right)$ in a FEL oscillator with a strong conventional guide field (solid line) and a reversed guide field for the same pitch factor of the electrons [dashed line in (a)].

resonance [compare solid and dashed lines in Fig. 15(a)]. This mechanism of efficiency enhancement is even more remarkable in an FEL-amplifier scheme [40]. Note also that the initial value of the parameter $\mu_{0}$ in the considered 
regime is negative (i.e. with the decrease in the electrons energy the axial velocity increases), and its optimal value $\mu_{0}^{\mathrm{opt}}$ increases with the increase in the rf-field amplitude [Fig. 15(b)]. We should also emphasize that a relatively high initial value of the bunching parameter is favorable for selfexcitation conditions and decreases the starting current of the FEL oscillator [2] [compare with (32) and (34)].

Note, however, that the strength of the axial guide magnetic field in the regime described above is rather close to the resonance value. This fact complicates the experimental realization of the FEL in such a regime. Obviously, the quality of the helical beam formation in this region of parameters requires the use of a longer tapered wiggler section. Simulations show that for the FEL parameters given in Fig. 13 a wiggler with ten periods of tapering provides an amplitude of parasitic oscillations around the steady-state trajectory lower than $5 \%$. This seems to be sufficient for experimental observation of FEL operation in the described regime. On the other hand, from relations (11) and (35), one can see that the value of guide field $f_{\mu=0}$ corresponding to $\mu=0$ is shifted from the cyclotron resonance value $f_{c r}$ (corresponding $\omega_{H}=\Omega_{b}$ ) with an increase in the electron energy (Fig. 16). Therefore, the described regime is more preferable for a FEL driven by strongly relativistic electron beams $\gamma \geq 5-10$.

Thus, theoretical analysis demonstrated potential for FEL efficiency enhancement in the conventional guide field regime when the gyrofrequency exceeds the bounce frequency using the effect of the dependence of the effective mass on energy of electrons oscillating in a wiggler and axial guide magnetic fields. However, for mildly relativistic electron energies $(\gamma \leq 2.5)$, the optimal strength of the guide magnetic field must be close to the resonance value and in this case it is extremely difficult to realize an adiabatic entrance of electrons in the wiggler field. The considered mechanism looks more applicable for strongly

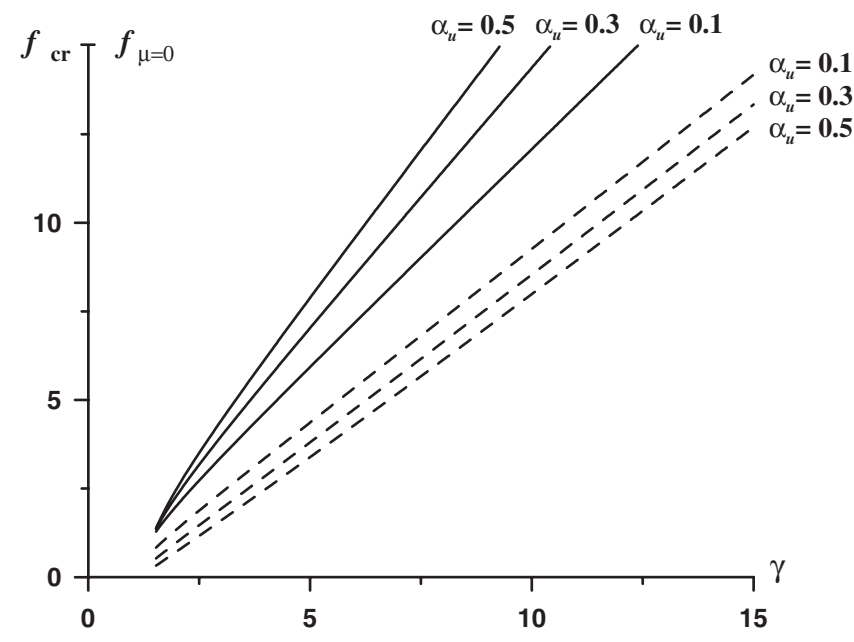

FIG. 16. The parameters $f_{c r}$ (dashed lines) and $f_{\mu=0}$, which correspond to the value $\mu=0$ (solid lines), as functions of electron energy $\gamma$ for different amplitudes of the wiggler field $\alpha_{w}$. relativistic electron beams $(\gamma \geq 5)$ when the optimal value of the guide magnetic field for such a regime is shifted from the cyclotron resonance.

\section{COMBINATION RESONANCES BETWEEN THE ELECTRONS AND THE RF WAVE. RADIATION UNDER ABNORMAL DOPPLER EFFECT}

For the combination resonances (3) it is useful to introduce the subtractive $\theta-\chi$ or the additive $\theta+\chi$ phases. In this case the electron-wave interaction may be described by the following equations:

$$
\begin{aligned}
& \frac{\mathrm{d} C}{\mathrm{~d} Z}=a \alpha_{s} \cos \varphi, \\
& \frac{\mathrm{d} \gamma}{\mathrm{d} Z}=-b \alpha_{s} \cos \varphi, \\
& \frac{\mathrm{d} \varphi}{\mathrm{d} Z}=g\left(\frac{\gamma}{p_{\| *}}-1\right)-1 \mp \Gamma+k C^{2}-\frac{a \alpha_{s}}{C} \sin \varphi,
\end{aligned}
$$

where $\varphi=\theta \mp \chi \mp \varphi_{C}$,

$$
\begin{aligned}
& a=\frac{1}{2 R}\left[\left(\frac{\gamma}{p_{\| *}}-1\right)(1 \mp R) \mp \frac{f \gamma p_{\perp *}^{2}}{p_{\| *}^{4} \Gamma}\right], \\
& b= \pm \frac{1}{2 p_{\| *}^{3}}\left[p_{\perp *}^{2}+p_{\| * *}^{2}(1 \mp R) \mp \frac{g \gamma p_{\perp *}^{2}}{p_{\| *} \Gamma}\right], \\
& k= \pm Q+\frac{g \gamma}{4 p_{\| * *}^{5}}\left[3 p_{\perp *}^{2}+p_{\| * *}^{2}\left(1+R^{2}\right)\right] .
\end{aligned}
$$

The upper sign in (44) and subsequent relations corresponds to the upper sign in the resonance conditions (3) while the lower one stands for the lower sign in (3) as well.

In the small-signal approximation $\alpha_{s} \rightarrow 0$ for electrons moving along the equilibrium helical trajectory when entering the interaction space [i.e. for the boundary conditions (A5a) and (A5b)] from Eq. (44) we get the expression for the linear efficiency,

$$
\eta_{\text {lin }}=\frac{\alpha_{s}^{2} L^{2}}{1-\gamma_{0}^{-1}} \frac{2 a_{0} b_{0}}{\gamma_{0}} \frac{\sin ^{2}[(\Gamma \mp \Delta) L]}{[(\Gamma \mp \Delta) L]^{2}},
$$

which coincides with results of linear theory for the FEL presented in [28]. Analysis of the coefficients $a_{0}$ and $b_{0}$ shows that the electrons which initially belong to group I orbits radiate energy $(\eta>0)$ under the conditions of resonance with upper sign in (3) and conversely absorb the rfwave energy $(\eta<0)$ when resonance with lower sign in (3) is fulfilled. In contrast, if the electrons move along the other stationary trajectory of group II orbits the radiation takes place under the lower sign in (3) while absorption under the resonance with upper sign.

For relatively small wiggler fields, when the frequency of electron oscillation near the equilibrium trajectory is given by Eq. (17), the resonance conditions (3) under which the radiation takes place can be written in a form which is universal for both groups of steady-state orbits: 


$$
\omega-h v_{\|} \approx 2 \Omega_{b}-\omega_{H}\left(1+\frac{p_{\perp 0}^{2}}{p_{\| 0}^{2}}\right) .
$$

Note that for the group I orbits this radiation can possess remarkably higher frequency than traditional wiggler radiation under the resonance (2). The absorption takes place for both groups of steady-state orbits under the resonance

$$
\omega-h v_{\|} \approx \omega_{H}\left(1+\frac{p_{\perp 0}^{2}}{p_{\| 0}^{2}}\right) .
$$

Evidently, when the wiggler field amplitude tends to zero $\left(\alpha_{w} \rightarrow 0\right)$, relation (46b) determines the frequencies while relation (45) gives the intensities of ordinary cyclotron absorption [2,32].

From Eqs. (44a) and (44b) one can see that these equations imply the integral of motion,

$$
C^{2}-C_{0}^{2}=-2 \int_{\gamma_{0}}^{\gamma} \frac{a}{b} \mathrm{~d} \gamma
$$

which for the case of the relatively small changes in electron energies $(|w| \ll 1)$ can be reduced as

$$
C^{2}-C_{0}^{2}=-\frac{2 a_{0}}{b_{0}}\left(\gamma-\gamma_{0}\right)
$$

According to Eq. (48), at $a_{0} / b_{0}>0$ electron deceleration is accompanied by the excitation of their oscillations near the equilibrium trajectory, while at $a_{0} / b_{0}<0$ we have a decrease of these oscillations with the decrease of the electron energy. Therefore, to obtain the radiation in the second case, the electrons before entering the interaction space have to move along the trajectory which is deferent from the equilibrium trajectory, i.e., the electrons have to possess an initial oscillation energy relative to the equilibrium trajectory $C_{0}^{2} \neq 0$. In the first case the radiation can be generated even when the electrons move along the equilibrium trajectory $C_{0}=0$. Evidently, the first case is typical for radiation under the abnormal Doppler effect while the second case for radiation under the normal Doppler effect [32]. The abnormal Doppler effect is observed under resonance conditions (46a) while the normal Doppler effect is realized at condition (46b).

Let us study here the nonlinear stage of interaction between the rf wave and the electrons moving in a regular wiggler section along the equilibrium helical trajectory under the synchronism (46a). Boundary conditions for Eq. (44) in this case are identical for all electrons in the beam:

$$
C\left(Z=Z_{0}\right)=0, \quad \gamma\left(Z_{0}\right)=0, \quad \varphi\left(Z_{0}\right)=0 .
$$

Thus, the excitation of the transverse oscillations around the steady-state trajectory and energy extraction is the same for all the beam electrons and the force bunching of the particles governed by the two last terms in the equation for the phase $\varphi$ (44c) dominate. Considering relatively small changes in the electrons energy and taking into account the integral of motion (48), we can reduce Eq. (44) to the form universal for the microwave generators based on the abnormal Doppler effect (compare with [2]):

$$
\frac{\mathrm{d} u}{\mathrm{~d} \varsigma}=\sqrt{u} \cos \varphi, \quad \frac{\mathrm{d} \varphi}{\mathrm{d} \varsigma}=\delta_{ \pm}+u \operatorname{sign} \nu-\frac{1}{2 \sqrt{u}} \sin \varphi
$$

with the boundary conditions $u(\varsigma=0)=0, \varphi(\varsigma=0)=0$. Here

$$
\begin{aligned}
u & =w \sqrt[3]{\frac{\nu^{2}}{2 a_{0} b_{0} \alpha_{s}^{2}}}, \quad \varsigma=\left(Z-Z_{0}\right) \sqrt[3]{2 a_{0} b_{0}|\nu| \alpha_{s}^{2}}, \\
\delta_{ \pm} & =\frac{1}{\sqrt[3]{2 a_{0} b_{0}|\nu| \alpha_{s}^{2}}}\left[g\left(\frac{\gamma_{0}}{p_{\| 0}}-1\right)-1 \mp \Gamma_{0}\right] .
\end{aligned}
$$

The parameter $\nu$ for the case of small wiggler fields $\alpha_{w} \ll 1$ is given by

$$
\nu=g\left[\frac{\gamma_{0}}{p_{\| 0}}(1 \mp 1)-1\right]+\frac{\gamma_{0}}{p_{\| 0}}\left[\frac{\gamma_{0}}{p_{\| 0}}(1 \pm 1)-1\right]+\frac{g \gamma_{0}^{3}}{p_{\| 0}^{3}} .
$$

The electron efficiency (with the identity of energy exchange with the wave for all the beam electrons taken into account) is determined by

$$
\eta=\frac{u}{1-\gamma_{0}^{-1}} \sqrt[3]{\frac{2 a_{0} b_{0} \alpha_{s}^{2}}{\nu^{2}}}
$$

Based on the integral of motion for Eq. (50),

$$
\sin \varphi=\delta_{ \pm} \sqrt{u}+\frac{u^{3 / 2}}{2} \operatorname{sign} \nu,
$$

we obtain (see [2] for more details) the maximum electron deceleration $u_{\max }=4$ is achieved at $\delta_{\mathrm{opt}}=-\frac{3}{2} \operatorname{sign} \nu$ and $\varphi=\frac{\pi}{2} \operatorname{sign} \nu$. In the regime of precise resonance $\delta_{ \pm}=0$, the maximum deceleration is $u^{\max }=\sqrt[3]{4}$ at $\varphi=\frac{\pi}{2} \operatorname{sign} \nu$. The optimal length of the oscillator in the last case is $\boldsymbol{s}_{\text {opt }}=3$.

Using analysis described above, let us estimate here the possibility of the FEL operation in the regime of combination resonance based on the linac LIU-3000. In the reversed guide field regime for the FEL parameters similar to ones given in Fig. 6 in the conditions of the resonance (46a) the radiation frequency would reach $100 \mathrm{GHz}$, i.e., for the same wiggler period and electron energy to be in 3 times higher than current operation frequency of the FEL. At the number of bounce oscillations over the interaction space $N \approx 5-6$ (which correspond to $\mathrm{s}_{\text {opt }}$ ) and the signal wave amplitude $\alpha_{s} \approx 0.4-0.5$ the electron efficiency would be at the level $\sim 5 \%-8 \%$. Thus, an important result of the above theoretical consideration is feasibility of regimes of combination resonances (3) for increasing the radiation frequency. In particular, these regimes allow advance of JINR-IAP FEL into the short part of the millimeter wavelength band keeping the electron beam energy and wiggler period. 


\section{CONCLUSION}

In the present paper, a nonlinear theory of an FEL with guide magnetic field is developed. The averaged equations for the e-beam/rf-wave interaction under traditional wiggler resonance (2) as well as for combination resonances (3) were obtained. This allowed the analysis of specific features of the e-beam/rf-wave interaction for different FEL operating regimes to be carried out. Our analysis demonstrates good agreement with some results of previous theoretical consideration (in the frame of average and nonaverage models) alongside some new effects including: (i) possibility of the FEL with guide magnetic field to operate at additional combination resonances which provide higher Doppler frequency up-conversion than in traditional wiggler resonance; (ii) possibility of efficiency enhancement when operating at the group of orbits II and utilizing dependence of the effective mass of electrons on their energy; (iii) explanation of the drop of FEL efficiency in conventional guide field orientation in comparison with reversed guide field regimes that is caused by a difference in electron beam quality after the tapered wiggler section; (iv) demonstration of advantages of a FEL with a reverse guide field in comparison with CARM to obtain high electron efficiency.

We should note also that in the present paper we used the 1D approximation for description of wiggler field and electron trajectories. Nevertheless, the approach suggested in this paper to derivation of the average motion equation is rather universal and can be implemented not only for the model of an ideal wiggler but for more sophisticated models of the real wigglers.

\section{ACKNOWLEDGMENTS}

Authors would like to thank Dr. A. K. Kaminsky, Dr. S.N. Sedykh (JINR, Dubna, Russia), Professor A.D. R. Phelps, Dr. A.W. Cross, Dr. C. G. Whyte (University of Strathclyde, Glasgow, U.K.) for useful discussions and joint experimental works with FELs, and Dr. S. V. Samsonov (IAP RAS, Nizhny Novgorod, Russia) for help in the work. This work is partially supported by the Russian Foundation for Basic Research.

\section{APPENDIX}

Let us find here the average equations for motion of magnetically guided electrons in the wiggler and rf field. Substituting (27) in Eq. (22) and assuming the deviations from the equilibrium trajectory caused by both initial oscillations and the influence of the signal wave as being relatively small $\left(\left|\rho_{1,2}\right| \ll\left|p_{\perp *}\right|\right)$, after the series expansion in $\rho_{1,2}$ of the right sides we can present Eq. (22) in the form

$$
\begin{aligned}
\frac{\mathrm{d} \rho_{1}}{\mathrm{~d} Z} & =\rho_{2}\left(1-\frac{f}{p_{\| *}}\right)-\rho_{2} f F_{1}\left(\rho_{1}, \rho_{2}\right)-\left(\frac{\gamma}{p_{\| *}}-1+\frac{\gamma p_{\perp *}}{p_{\| *}^{3}} \rho_{1}\right) \alpha_{s} \sin \theta-\frac{\mathrm{d} p_{\perp *}}{\mathrm{~d} \gamma} \frac{\mathrm{d} \gamma}{\mathrm{d} Z}, \\
\frac{\mathrm{d} \rho_{2}}{\mathrm{~d} Z} & =-\rho_{1}\left(1-\frac{f}{p_{\| *}}-\frac{f p_{\perp *}^{2}}{p_{\| *}^{3}}\right)+\rho_{1} f F_{1}\left(\rho_{1}, \rho_{2}\right)+p_{\perp *} f F_{2}\left(\rho_{1}, \rho_{2}\right)+\left(\frac{\gamma}{p_{\| *}}-1+\frac{\gamma p_{\perp *}}{p_{\| * *}^{3}} \rho_{1}\right) \alpha_{s} \cos \theta, \\
\frac{\mathrm{d} \gamma}{\mathrm{d} Z} & =-\frac{\alpha_{s}}{p_{\| *}}\left\{\left[p_{\perp *}+\rho_{1}\left(1+\frac{p_{\perp *}^{2}}{p_{\| *}^{2}}\right)\right] \sin \theta-\rho_{2} \cos \theta\right\}, \\
\frac{\mathrm{d} \theta}{\mathrm{d} Z} & =g\left(\frac{\gamma}{p_{\| *}}-1\right)-1+g \gamma F_{1}\left(\rho_{1}, \rho_{2}\right) .
\end{aligned}
$$

The functions $F_{1,2}$ in (A1) describe the unharmonic (nonisochronous) nature of electron oscillations near the equilibrium trajectory and can be written as

$$
\begin{aligned}
& F_{1}=\frac{1}{p_{\| * *}^{3}}\left\{p_{\perp *} \rho_{1}+\frac{1}{2}\left[\rho_{1}^{2}\left(1+\frac{3 p_{\perp *}^{2}}{p_{\| *}^{2}}\right)+\rho_{2}^{2}\right]\right\}, \\
& F_{1}=\frac{1}{2 p_{\| * *}^{3}}\left[\rho_{1}^{2}\left(1+\frac{3 p_{\perp *}^{2}}{p_{\| *}^{2}}\right)+\rho_{2}^{2}+\frac{3 p_{\perp *}}{p_{\| *}^{2}} \rho_{1} \rho_{2}^{2}+\rho_{1}^{3}\left(\frac{3 p_{\perp *}}{p_{\| *}^{2}}+\frac{5 p_{\perp *}^{3}}{p_{\| *}^{4}}\right)\right] .
\end{aligned}
$$

Obviously, Eq. (A1) describes a nonlinear oscillator which is affected by the external rf wave. At $\alpha_{s}=0$ after linearization these equations are reduced to Eq. (13). Thus, at $\alpha_{s} \neq 0$ using a method of variations of arbitrary constants, the solution of Eq. (A1) can be presented as

$$
\rho_{1}=C_{1}(Z) \sin \chi+C_{2}(Z) \cos \chi, \quad \rho_{1}=R\left[C_{1}(Z) \cos \chi-C_{2}(Z) \sin \chi\right],
$$

where $\chi=\int_{\mathrm{Z}_{0}}^{\mathrm{Z}} \Gamma \mathrm{d} Z, R=\sqrt{\left(1-\frac{f}{p_{\| *}}-\frac{f p_{\perp *}^{2}}{p_{\| *}^{3}}\right) /\left(1-\frac{f}{p_{\| *}}\right)}$. After substitution of (A3), Eq. (A1) can be reduced to the form which facilitates subsequent averaging: 


$$
\begin{aligned}
\mathrm{e}^{\mathrm{i} \chi} \frac{\mathrm{d} C_{+}}{\mathrm{d} Z}= & -\mathrm{i} \alpha_{s}\left(\frac{\gamma}{p_{\| *}}-1+\frac{\gamma p_{\perp *}}{p_{\| *}^{3}}\left(C_{1} \sin \chi+C_{2} \cos \chi\right)\right)\left(\sin \theta+\mathrm{i} R^{-1} \cos \theta\right)-\mathrm{i} \frac{\mathrm{d} p_{\perp *}}{\mathrm{~d} \gamma} \frac{\mathrm{d} \gamma}{\mathrm{d} Z} \\
& +f R^{-1}\left\{p_{\perp *} F_{2}\left(C_{+}, \chi\right)+F_{1}\left(C_{+}, \chi\right)\left[C_{1}\left(\sin \chi-\mathrm{i} R^{2} \cos \chi\right)+C_{2}\left(\cos \chi+\mathrm{i} R^{2} \sin \chi\right)\right]\right\} \\
& -\frac{1}{R} \frac{\mathrm{d} R}{\mathrm{~d} \gamma} \frac{\mathrm{d} \gamma}{\mathrm{d} Z}\left(C_{1} \cos \chi-C_{2} \sin \chi\right), \\
\frac{\mathrm{d} \gamma}{\mathrm{d} Z}= & -\frac{\alpha_{s}}{p_{\| *}}\left\{\left[p_{\perp *}+\left(1+\frac{p_{\perp *}^{2}}{p_{\| *}^{2}}\right)\left(C_{1} \sin \chi+C_{2} \cos \chi\right)\right] \sin \theta-R\left(C_{1} \cos \chi-C_{2} \sin \chi\right) \cos \theta\right\}, \\
\frac{\mathrm{d} \theta}{\mathrm{d} Z}= & g\left(\frac{\gamma}{p_{\| *}}-1\right)-1+g \gamma F_{1}\left(C_{+}, \chi\right),
\end{aligned}
$$

where $C_{+}=C_{1}+\mathrm{i} C_{2}$. For a monoenergetic unmodulated electron beam boundary conditions to Eq. (A3) have a form

$$
\gamma\left(Z_{0}\right)=\gamma_{0}, \quad \theta\left(Z_{0}\right)=\theta_{0} \in[0,2 \pi)
$$

and

$$
C_{1,2}\left(Z=Z_{0}\right)=0
$$

for the electrons moving along the helical steady-state trajectory at the entrance of the interaction space while

$$
C_{1,2}\left(Z=Z_{0}\right)=C_{01,2}
$$

for the electrons oscillating near this trajectory.

\section{Wiggler resonance between the electrons and the rf wave}

When the electrons interact with the rf wave under the condition of the wiggler resonance (2), the phase $\theta$ is a slow function of $z$ while the phase $\chi$ exhibits fast oscillations if the condition (1) is fulfilled. This allows Eq. (A4) to be averaged over the phase $\chi$. As a result, Eq. (A4a) acquires the form

$$
\begin{aligned}
\frac{\mathrm{d} \bar{C}_{+}}{\mathrm{d} Z}= & \frac{\alpha_{s} \bar{C}_{+}}{2 p_{\| *}}\left\{\frac{\mathrm{d} p_{\perp *}}{\mathrm{~d} \gamma}\left[\left(1+\frac{p_{\perp *}^{2}}{p_{\| *}^{2}}\right) \sin \bar{\theta}-i R \cos \bar{\theta}\right]\right. \\
& \left.+\left(\frac{p_{\perp *}}{R} \frac{\mathrm{d} R}{\mathrm{~d} \gamma}-\frac{\gamma p_{\perp *}}{p_{\| *}^{2}}\right) \sin \bar{\theta}-\mathrm{i} \frac{\bar{\gamma} p_{\perp *}}{p_{\| *}^{2} R} \cos \bar{\theta}\right\} \\
& +\frac{3 f\left(\bar{C}_{+} \bar{C}_{1} \bar{C}_{2}-\mathrm{i} \bar{C}_{1}^{3}+\bar{C}_{2}^{3}\right)}{16 R p_{\| * *}^{3}}\left(1+\frac{6 p_{\perp *}^{2}}{p_{\| *}^{2}}+\frac{5 p_{\perp *}^{4}}{p_{\| *}^{4}}\right. \\
& \left.+\frac{2 R^{2}}{3}\left(1+\frac{3 p_{\perp *}^{2}}{p_{\| *}^{2}}\right)+R^{4}\right),
\end{aligned}
$$

where "-" is the sign of averaging. To get the correct average equation for the slow phase $\theta$, one has to include in the consideration the oscillatory component of the transverse momentum:

$\tilde{C}_{+}=\frac{\alpha_{s}}{\Gamma}\left[\left(\frac{\gamma}{p_{\| *}}-1\right)\left(\sin \theta+\mathrm{i} R^{-1} \cos \theta\right)-\frac{\mathrm{d} p_{\perp *}}{\mathrm{~d} \gamma} \frac{p_{\perp *}}{p_{\| *}} \sin \theta\right] \mathrm{e}^{\mathrm{i} \chi}$.
Substituting into Eqs. (A4b) and (A4c) the transverse momentum as a sum of the average component (A6) and the oscillatory component (A7) $C_{+}=\bar{C}_{+}+\tilde{C}_{+}$, after averaging we get [29]

$$
\begin{aligned}
\frac{\mathrm{d} \bar{\gamma}}{\mathrm{d} Z}= & -\frac{\alpha_{s} p_{\perp *}}{p_{\| *}} \sin \bar{\theta}, \\
\frac{\mathrm{d} \bar{\theta}}{\mathrm{d} Z}= & g\left(\frac{\bar{\gamma}}{p_{\| *}}-1\right)-1+\frac{\alpha_{s} \bar{\gamma} p_{\perp *}}{p_{\| * *}^{3} R \Gamma} \cos \bar{\theta} \\
& +\frac{g \bar{\gamma}}{4 p_{\| * *}^{3}}\left[1+R^{2}+\frac{3 p_{\perp *}^{2}}{p_{\| *}^{2}}\right]\left(\bar{C}_{1}^{2}+\bar{C}_{2}^{2}\right) .
\end{aligned}
$$

Note that the oscillatory component of the transverse momentum $\tilde{C}_{+}$leads to the appearance of the term proportional to $\alpha_{s}$ in the equation for the slow phase (A8b) and should be taken into account. At the same time, the same oscillatory component results in the presence of the term proportional to $\alpha_{s}^{2}$ in the equation for energy (A8a) and, therefore, can be neglected.

As seen from Eq. (A8), the energy variation depends on the value of $\left|\bar{C}_{+}\right|^{2}$. For this variable from Eq. (A6) we have

$$
\begin{aligned}
\frac{\mathrm{d}\left|C_{+}\right|^{2}}{\mathrm{~d} Z}= & \left|\bar{C}_{+}\right|^{2} \frac{\alpha_{s} p_{\perp *}}{p_{\| *}}\left\{p_{\perp *}^{-1} \frac{\mathrm{d} p_{\perp *}}{\mathrm{~d} \gamma}\left(1+\frac{p_{\perp *}^{2}}{p_{\| *}^{2}}\right)\right. \\
& \left.+R^{-1} \frac{\mathrm{d} R}{\mathrm{~d} \gamma}-\frac{\gamma}{p_{\| *}^{2}}\right\} \sin \theta .
\end{aligned}
$$

The equations (A8a) and (A9) possess the motion integral, which in the case of relatively small transverse momentum $p_{\perp *}^{2} \ll p_{\| *}^{2}$ may be presented as

$$
\frac{\left|\bar{C}_{+}\right|^{2} p_{\perp *} R}{\sqrt{\gamma^{2}-1}}=\mathrm{const}=\frac{\left|\bar{C}_{+0}\right|^{2} p_{\perp 0} R_{0}}{\sqrt{\gamma_{0}^{2}-1}},
$$

where $\bar{C}_{+0}=\bar{C}_{+}\left(Z=Z_{0}\right)$ is the initial value of the small oscillations of the electrons around the steady-state helical trajectory [compare with $(\mathrm{A} 5 \mathrm{c})]$. Taking into account the motion integral (A10), the average motion equations (A8) take the form (28). 


\section{Combination resonances between the electrons and the rf wave}

When the combination resonances (3) are realized the subtractive $\theta-\chi$ or the additive $\theta+\chi$ phase becomes slow. In this case, averaging Eq. (A4) over the rapid phases $\theta$ and $\chi$ and introducing a polar coordinates $C=\sqrt{C_{1}^{2}+C_{2}^{2}}, \varphi_{C}=\operatorname{arctg}\left(C_{1} / C_{2}\right)$ we get (44).

[1] R. K. Parker, R. H. Jackson, S. H. Gold, H. P. Freund, V. L. Granatstein, P.C. Efthimion, M. Herndon, and A.K. Kinkead, Phys. Rev. Lett. 48, 238 (1982).

[2] V.L. Bratman, G. G. Denisov, N. S. Ginzburg, and M. I. Petelin, IEEE J. Quantum Electron. 19, 282 (1983).

[3] S. H. Gold, D. L. Hardesty, A. K. Kinkead, L. R. Barnett, and V. L. Granatstein, Phys. Rev. Lett. 52, 1218 (1984).

[4] J. Masud, T. C. Marshall, S. P. Schlesinger, and F. G. Yee, Phys. Rev. Lett. 56, 1567 (1986).

[5] F. Hartemann, K. Xu, G. Bekefi, J.S. Wurtele, and J. Fajans, Phys. Rev. Lett. 59, 1177 (1987).

[6] A. A. Kaminsky, A. K. Kaminsky, and S. B. Rubin, Part. Accel. 33, 189 (1990).

[7] M. E. Conde and G. Bekefi, Phys. Rev. Lett. 67, 3082 (1991).

[8] M. Wang, Z. Wang, J. Chen, Z. Lu, and L. Zhang, Nucl. Instrum. Methods Phys. Res., Sect. A 304, 116 (1991).

[9] N. Ikeda, K. Okubo, A. Zakou, S. Ishii, R. Matsuda, T. Yamanaka, S. Kuribayashi, T. Akiba, S. Nakai, N. Ohigashi, J. Chen, K. Imasaki, and C. Yamanaka, Nucl. Instrum. Methods Phys. Res., Sect. A 331, 130 (1993).

[10] T. S. Chu, F. Hartemann, B. G. Danly, and R. J. Temkin, Phys. Rev. Lett. 72, 2391 (1994).

[11] P. Zambon, W. J. Witteman, and P. J. M. Van der Slot, Nucl. Instrum. Methods Phys. Res., Sect. A 341, 88 (1994).

[12] A. W. Cross, N. S. Ginzburg, W. He, D. A. Jaroszynski, N. Yu. Peskov, A. D. R .Phelps, and C. G. Whyte, Nucl. Instrum. Methods Phys. Res., Sect. A 407, 181 (1998).

[13] A. V. Arzhannikov, N. S. Ginzburg, V. G. Ivanenko, I. A. Ivanov, P. V. Kalinin, A. S. Kuznetsov, S. A. Kuznetsov, N. Yu. Peskov, A. S. Sergeev, S. L. Sinitsky, V.D. Stepanov, and V. Yu. Zaslavsky, JETP Lett. 87, 715 (2008).

[14] A. V. Arzhannikov, A. W. Cross, N. S. Ginzburg, W. He, P. V. Kalinin, I. V. Konoplev, S. A. Kuznetsov, N. Yu. Peskov, A. D. R. Phelps, C. W. Robertson, K. Ronald, A. S. Sergeev, S. L. Sinitsky, V. D. Stepanov, M. Thumm, C. G. Whyte, and V. Yu. Zaslavsky, IEEE Trans. Plasma Sci. 37, 1792 (2009).

[15] I. V. Konoplev, P. McGrane, W. He, A. W. Cross, A. D. R. Phelps, C. G. Whyte, K. Ronald, and C. W. Robertson, Phys. Rev. Lett. 96, 035002 (2006).

[16] I. V. Konoplev, A. W. Cross, A. D. R. Phelps, W. He, K. Ronald, C. G. Whyte, C. W. Robertson, P. MacInnes, N. S. Ginzburg, N. Yu. Peskov, A. S. Sergeev, V. Yu. Zaslavsky, and M. Thumm, Phys. Rev. E 76, 056406 (2007).
[17] N. S. Ginzburg, A. A. Kaminsky, A. K. Kaminsky, N. Yu. Peskov, S. N. Sedykh, A. P. Sergeev, and A. S. Sergeev, Phys. Rev. Lett. 84, 3574 (2000).

[18] A. K. Kaminsky, E. A. Perelshtein, S. N. Sedykh, N. S. Ginzburg, S. V. Kuzikov, N. Yu. Peskov, and A. S. Sergeev, Tech. Phys. Lett. 36, 37 (2010).

[19] N. S. Ginzburg, I. I. Golubev, A. K. Kaminsky, A.P. Kozlov, S. V. Kuzikov, E. A. Perelshtein, N. Yu. Peskov, M. I. Petelin, S. N. Sedykh, A. P. Sergeev, A. S. Sergeev, A. A. Vikharev, and N.I. Zaitsev, Phys. Rev. ST Accel. Beams 14, 041002 (2011).

[20] L.Friedland, Phys. Fluids 23, 2376 (1980).

[21] A. M. Kondratenko and E. L. Saldin, Zh. Tekh. Fiz. 51, 1633 (1981) (in Russian).

[22] H. P. Freund, P. Sprangle, I. Dillenburg, E. H. Jornada, B. Liberman, and R.S. Schneider, Phys. Rev. A 24, 1965 (1981).

[23] H. P. Freund and A. T. Drobot, Phys. Fluids 25, 736 (1982).

[24] H. P. Freund, S.Johnston, and P. Sprangle, IEEE J. Quantum Electron. 19, 322 (1983).

[25] H.P. Freund and A. K. Ganguly, IEEE J. Quantum Electron. 21, 1073 (1985).

[26] H. P. Freund, Phys. Fluids B 5, 1869 (1993).

[27] H.P. Freund and T.M. Antonsen, Principles of FreeElectron Lasers (Chapman \& Hall, London, 1996), 2nd ed.

[28] N. S. Ginzburg and Yu. V. Novozhilova, Zh. Tekh. Fiz. 56, 1709 (1986) (in Russian).

[29] N. S. Ginzburg and N. Yu. Peskov, Zh. Tekh. Fiz. 58, 859 (1988) (in Russian).

[30] V. Goryashko, K. Ilyenko, and A. Opanasenko, Phys. Rev. ST Accel. Beams 12, 100701 (2009).

[31] X. Huang, S. Wang, Y. Xu, and S. Zhang, Phys. Rev. ST Accel. Beams 15, 120702 (2012).

[32] V. L. Ginzburg and I. M. Frank, Doklady AS USSR 56, 583 (1947) (in Russian).

[33] V. K. Yulpatov, in Gyrotron, edited by A. V. GaponovGrekhov (IAP AS USSR, Gorky, USSR, 1981), p. 26 (in Russian).

[34] G. S. Nusinovich, Introduction to physics of gyrotrons (J. Hopkins University Press, Baltimore, Maryland, 2004).

[35] A. K. Kaminsky, A. A. Kaminsky, S. N. Sedykh, and A. P. Sergeev, in Proceedings of the 18th International FEL Conference (Elsevier Science B.V., Rome, Italy, 1996), p. 109.

[36] N. Yu. Peskov, S. V. Samsonov, N. S. Ginzburg, and V. L. Bratman, Nucl. Instrum. Methods Phys. Res., Sect. A 407, 107 (1998).

[37] N. S. Ginzburg, A. K. Kaminsky, A. A. Kaminsky, N. Yu. Peskov, S. N. Sedykh, A.P. Sergeev, and A.S. Sergeev, IEEE Trans. Plasma Sci. 26, 536 (1998).

[38] V. L. Bratman, G. G. Denisov, B. D. Kol'chugin, S. V. Samsonov, and A. B. Volkov, Phys. Rev. Lett. 75, 3102 (1995).

[39] G.Bekefi, A. DiRienzo, C. Leibovitch, and B. G. Danly, Appl. Phys. Lett. 54, 1302 (1989.

[40] N. S. Ginzburg and N. Yu. Peskov, Zh. Tekh. Fiz. 61, 124 (1991) (in Russian) [Sov. Tech. Phys. 36, 1162 (1991)]. 